\title{
Perfectly matched layers for the Dirac equation in general electromagnetic texture
}

\author{
Walter Pötz \\ Institut für Physik, Karl-Franzens-Universität Graz, Universitätsplatz 5, 8010 Graz, Austria
}

(Dated: May 28, 2021)

Perfectly matched layer (PML) boundary conditions are constructed for the Dirac equation and general electromagnetic potentials. A PML extension is performed for the partial differential equation and two versions of a staggered-grid single-cone finite-difference scheme. For the latter, PML auxiliary functions are computed either within a Crank-Nicholson scheme or one derived from the formal continuum solution in integral form. Stability conditions are found to be more stringent than for the original scheme. Spectral properties under spatially uniform PML confirm damping of any out-propagating wave contributions. Numerical tests deal with static and time-dependent electromagnetic textures in the boundary regions for parameters characteristic for topological insulator surfaces. When compared to the alternative imaginary-potential method, PML offers vastly improved wave absorption owing to a more efficient suppression of back-reflection. Remarkably, this holds for time-dependent textures as well, making PML a useful approach for transient transport simulations of Dirac fermion systems.

PACS numbers: 05.60.Gg,02.30.Jr, 02.60.Lj,03.65.Pm,72.80.Vp,72.25.?b 


\section{INTRODUCTION}

The Dirac equation is a partial differential equation (PDE) which captures the relativistic quantum dynamics of (single) spin-1/2 particles [1, 2]. It is an integral part of the standard model of particle physics and finds application in atomic, molecular, condensed matter, and astrophysics alike [3 5. In solid-state physics, the Dirac equation in $(2+1) \mathrm{D}$ (one time and two space dimensions) provides an effective model for the low-energy metallic surface excitations of graphene and topological insulators [6, 7].

Although there exist analytic solutions to the Dirac equation in electromagnetic fields, in most applications the solution must be sought numerically [8]. This makes the development of efficient and spectrally accurate numerical approaches desirable. The present efforts have been triggered by the discovery of topological insulator surface states and their potential for structural design [6, 7]. Our aim is the development of an efficient computational framework for the description of Dirac fermion dynamics on the surface of topological insulators. We have recently developed single-cone finite-difference schemes (FDS) for the numerical treatment of the time-dependent Dirac equation 9 . They are explicit, second-order accurate and, via an identified conserved functional, allow an analytic analysis of stability. The underlying multi-component leap-frog scheme yields single-cone lattice schemes. In other words, there is no fermion doubling on the grid. For details we refer the reader to earlier work [9, 10. Since this FDS does not lead to any spurious (counter-propagating) doublers, perfectly-matched layers PMLs for the FDS can be inferred from the continuum Dirac equation. This has been demonstrated by Pinaud in recent work 11. Indeed, the construction of absorbing, transparent, or open boundary conditions (BCs) is an essential part of any numerical treatment of open quantum systems, such as quantum transport or particle-scattering simulations. A review and comparison of BCs for quantum wave equations can be found in the literature [12. Recently, this PML formulation has been joined with a pseudo-spectral method for the computation of the time-dependent Dirac equation [13, 14].

The skeleton FDS has recently been extended to incorporate general electromagnetic textures [15. The latter have a profound influence on the electronic structure and transport properties of TI surfaces 6, 7]. The aim of this work is the construction of PML boundary conditions in presence of electromagnetic texture in the boundary layers. Several variants of a PML extension of the single-cone FDS will be discussed.

The paper is organised as follows. In Sect. II PML is formulated for the Dirac equation with general electromagnetic potentials in the absorbing layers. PML-induced spectral modifications are explored. In Sect. III these results are used to construct PML schemes for the single-cone FDS of the Dirac equation. Several second-order accurate methods for the computation of the arising PML auxiliary functions are discussed. In Sect. IIIB we present a PML extension for a FDS where the scalar potential term is added directly, whereas the vector potential is introduced via a Peierls-Schwinger phase factor. In Sect. III C PML is constructed for a gauge-invariant FDS where the entire electromagnetic potential is introduced by Schwinger substitution. Spectral differences arising from these two formulations are discussed in Sect. IVA. Furthermore, stability conditions of the PML extended FDSs are derived in Sect. IV. Numerical examples and a comparison to the imaginary-potential method (IPM) are given in Sect. V Sects. V A. $\mathrm{VB}$, and V C deal with time-independent textures. Time-dependent textures are studied in Sect. V D. Summary and Conclusions are given in Sect VI.

\section{PML FOR THE CONTINUUM DIRAC EQUATION (PDE)}

Aiming at simulations of Dirac fermion transport on topological insulator (TI) surfaces, under open BCs, we develop PML for the $(2+1)$ D Dirac equation in the TI representation. Thereby the efficiency of PML for FDS in more than one space dimensions will be exposed. An extension to the $(3+1) \mathrm{D}$ form, e.g. for a simulation of relativistic electrons, is straight-forward.

The free-particle Dirac equation in Schrödinger form for the two-component spinor $\psi=(u, v)$ reads

$$
i \hbar \partial_{t}\left(\begin{array}{l}
u(x, y, t) \\
v(x, y, t)
\end{array}\right)=\left(\begin{array}{cc}
m & c \frac{\hbar}{i}\left(\partial_{y}+i \partial_{x}\right) \\
c \frac{\hbar}{i}\left(\partial_{y}-i \partial_{x}\right) & -m
\end{array}\right)\left(\begin{array}{l}
u(x, y, t) \\
v(x, y, t)
\end{array}\right)
$$

$m$ represents the mass term (with the speed of light $c_{o}$ absorbed in $m \geq 0$ ). $c>0$ denotes the phase velocity, with $c=c_{o}$ for relativistic electrons [1].

An electromagnetic potential may be introduced via the Schwinger phase factor [16]. The $U(1)$ Schwinger substitution for the spinor $\psi$ is defined as follows [9, 10, 16]:

$$
\psi(x, y, t) \rightarrow \tilde{\psi}(x, y, t)=\exp \{i a(x, y, t)\} \psi(x, y, t)
$$

The real-valued phase $a(x, y, t)$ is defined as the line integral of the 3 -vector potential $(\mathcal{A}, \Phi)$ along $\left(\mathbf{s}, s_{o}\right)$ in 3 -vector 
(Minkowski) space. Here, $s_{x}=x, s_{y}=y, s_{o}=c t$.

$$
a(x, y, t)=\frac{1}{\hbar c_{o}} \int_{\left(x_{o}, y_{o}, c t_{o}\right)}^{(x, y, c t)}\left[d \mathbf{s}^{\prime} \cdot e \mathcal{A}\left(\mathbf{s}^{\prime}, s_{o}^{\prime} / c\right)+\frac{c_{o}}{c} d s_{o}^{\prime} V\left(\mathbf{s}^{\prime}, s_{o}^{\prime} / c\right)\right] .
$$

Here $q=-e$ for the electron charge, and we set $V=-e \Phi$. The Dirac PDE in presence of electromagnetic potentials takes the form

$$
i \hbar \partial_{t}\left(\begin{array}{l}
u(x, y, t) \\
v(x, y, t)
\end{array}\right)=\left(\begin{array}{cc}
V(x, y, t)+m(x, y, t) & c\left(\hat{\Pi}_{y}(x, y, t)+i \hat{\Pi}_{x}(x, y, t)\right) \\
c\left(\hat{\Pi}_{y}(x, y, t)-i \hat{\Pi}_{x}(x, y, t)\right) & V(x, y, t)-m(x, y, t)
\end{array}\right)\left(\begin{array}{c}
u(x, y, t) \\
v(x, y, t)
\end{array}\right) .
$$

$\hat{\Pi}_{j}=\hbar / i \partial_{j}+e / c_{o} \mathcal{A}_{j}(x, y, t), j=x, y$ are the components of the kinetic momentum operator in the real-space representation. Note that the path-dependent local phase factor $\exp \{i a(x, y, t)\}$ was removed after partial derivatives of the spinor components were performed.

The formulation of PML is founded upon plane-wave solutions to Eq. (4) with a normal incidence on the simulation boundary. Position is continued into the complex plane so that all out-propagating wave contributions are damped [11, 17. The correct sign of the imaginary part may be determined by the ratio between group velocity and k-vector along the direction of incidence [18]. For constant electromagnetic potentials the energy dispersion is

$$
E=\hbar \omega= \pm\left[c^{2} \boldsymbol{\Pi}(k)^{2}+m^{2}\right]^{1 / 2}+V, \boldsymbol{\Pi}(k)=\hbar \mathbf{k}+\frac{e}{c_{o}} \mathcal{A}
$$

with the group velocity given by

$$
\mathbf{v}\left(\Pi_{x}, \Pi_{y}\right)=\frac{c^{2} \Pi(k)}{\hbar \omega-V}
$$

Following a general formulation, the PML substitution for propagation along $x$ reads [18]

$$
\Pi_{x}(k) x \rightarrow \Pi_{x}(k)\left(x+i \gamma(x) \frac{v_{x}\left(\Pi_{x}, \Pi_{y}\right)}{\Pi_{x}}\right)=\Pi_{x}(k)\left(x+i \gamma(x) \frac{c^{2}}{\hbar \omega-V}\right), \gamma(x) \geq 0 .
$$

Thereby the sign of the group velocity determines the complex $x$-coordinate extension into either the upper or lower complex half-plane so that all out-propagating wave contributions are damped.

The PML transform for the PDE is best determined in two steps: first consider zero electromagnetic potentials, then perform the Schwinger substitution. We shall use the notation $x_{j}=x, y$ for, respectively, $j=x, y$ [9] 15 . Setting $x_{j}^{\prime}=x_{j}+i \gamma^{j}\left(x_{j}\right) \frac{c^{2}}{\hbar \omega}$ the (pseudo-)differential form of the PML substitution is

$$
\partial_{x} \rightarrow \partial_{x^{\prime}}=\frac{1}{1+\frac{\sigma^{x}(x)}{\partial_{t}}} \partial_{x}=\partial_{x}-\hat{D}_{x}, \quad \hat{D}_{x}=\frac{\sigma^{x}(x)}{\partial_{t}+\sigma^{x}(x)} \partial_{x}
$$

and

$$
\partial_{y} \rightarrow \partial_{y^{\prime}}=\frac{1}{1+\frac{\sigma^{y}(y)}{\partial_{t}}} \partial_{y}=\partial_{y}-\hat{D}_{y}, \quad \hat{D}_{y}=\frac{\sigma^{y}(y)}{\partial_{t}+\sigma^{y}(y)} \partial_{y}
$$

The PML damping functions $\sigma^{j}(x) \equiv c^{2} d \gamma^{j}\left(x_{j}\right) / d x_{j}(\geq 0)$ are zero in the interior domain of the computation region and grow along $x_{j}$ when approaching the computation boundary [19]. This essentially is the substitution proposed by Pinaud [1].

It is useful to introduce

$$
D_{j}(x, y, t) \equiv \hat{D}_{j}\left(\begin{array}{c}
v(x, y, t) \\
u(x, y, t)
\end{array}\right), D_{j}(x, y, 0)=\left(\begin{array}{l}
0 \\
0
\end{array}\right), j=x, y .
$$

Note the order of the spinor components. Under Schwinger substitution $D_{j}(x, y, t)$ is transformed into $\tilde{D}_{j}(x, y, t)=$ $e^{-i a(x, y, t)} \hat{D}_{j}(x, y, t)\left(e^{i a(x, y, t)} \psi\right)$, where the factor $e^{-i a(x, y, t)}$ arises from the subtraction of the local phase, as in Eq. (4). Moving the Schwinger factor $e^{i a}$ to the left, $\tilde{D}_{j}$ may be recast

$$
\tilde{D}_{j}=e^{-i a} \hat{D}_{j} \tilde{\psi}=e^{-i a} \frac{\sigma^{j}\left(x_{j}\right)}{\partial_{t}+\sigma^{j}\left(x_{j}\right)} \partial_{x_{j}}\left(e^{i a} \psi\right)=\frac{\sigma^{j}\left(x_{j}\right)}{\partial_{t}+i V / \hbar+\sigma^{j}\left(x_{j}\right)}\left(\partial_{x_{j}}+\frac{i e}{\hbar c_{o}} \mathcal{A}_{j}\right) \psi
$$


Introducing

$$
V_{I}^{x}(x)=-i \hbar \sigma^{x}(x), \quad V_{I}^{y}(y)=-i \hbar \sigma^{y}(y),
$$

one obtains

$$
\begin{aligned}
& \left(i \hbar \partial_{t}-V-V_{I}^{x}(x)\right) \tilde{D}_{x}=-\frac{i}{\hbar} V_{I}^{x}(x) \hat{\Pi}_{x}\left(\begin{array}{c}
v \\
u
\end{array}\right) \\
& \left(i \hbar \partial_{t}-V-V_{I}^{y}(y)\right) \tilde{D}_{y}=-\frac{i}{\hbar} V_{I}^{y}(y) \hat{\Pi}_{y}\left(\begin{array}{c}
v \\
u
\end{array}\right) .
\end{aligned}
$$

For the FDS below, it will be convenient to start from

$$
\left(\partial_{t}+i \frac{V_{I}^{j}\left(x_{j}\right)}{\hbar}\right)\left(e^{i a} \tilde{D}_{j}\right)=i \frac{V_{I}^{j}\left(x_{j}\right)}{\hbar} \partial_{x_{j}}\left(e^{i a} \psi\right), D_{j}(0)=0
$$

In summary, the PML transformed PDE for the entire computation domain reads

$$
\begin{aligned}
i \hbar \partial_{t}\left(\begin{array}{c}
u(x, y, t) \\
v(x, y, t)
\end{array}\right) & =\left(\begin{array}{cc}
V(x, y, t)+m(x, y, t) & c\left(\hat{\Pi}_{y}(x, y, t)+i \hat{\Pi}_{x}(x, y, t)\right) \\
c\left(\hat{\Pi}_{y}(x, y, t)-i \hat{\Pi}_{x}(x, y, t)\right) & V(x, y, t)-m(x, y, t)
\end{array}\right)\left(\begin{array}{c}
v(x, y, t) \\
u(x, y, t)
\end{array}\right) \\
& -c \frac{\hbar}{i}\left(\begin{array}{cc}
0 & \left.\tilde{D}_{y}(x, y, t)+i \tilde{D}_{x}(x, y, t)\right) \\
\tilde{D}_{y}(x, y, t)-i \tilde{D}_{x}(x, y, t) & 0
\end{array}\right) .
\end{aligned}
$$

The last matrix represents a dissipator. It destroys unitarity under time-evolution, as generated by the Hamiltonian of the original Dirac equation (4) under suitable (e.g. zero or periodic) BCS. We note that subsystem quantum dynamics is non-unitary and non-norm-preserving. Here it is the openness of the system, i.e. the fact that the particle may leave or enter the simulation region (the subsystem), which brings about non-unitarity. PML overwrites zero or periodic BCs providing a proper subsystem dynamics.

The dissipator matrix elements may be computed from Eqs. (13) and (14). In integral form they read

$$
\begin{aligned}
& \tilde{D}_{x}(x, y, t)=-\frac{1}{\hbar^{2}} \int_{0}^{t} d \tau e^{\frac{i}{\hbar}\left(V(x, y, \tau)+V_{I}^{x}(x)\right)(\tau-t)} V_{I}^{x}(x) \Pi_{x}(x, y, \tau)\left(\begin{array}{c}
v(x, y, \tau) \\
u(x, y, \tau)
\end{array}\right) \\
& \tilde{D}_{y}(x, y, t)=-\frac{1}{\hbar^{2}} \int_{0}^{t} d \tau e^{\frac{i}{\hbar}\left(V(x, y, \tau)+V_{I}^{y}(y)\right)(\tau-t)} V_{I}^{y}(y) \Pi_{x}(x, y, \tau)\left(\begin{array}{c}
v(x, y, \tau) \\
u(x, y, \tau)
\end{array}\right) .
\end{aligned}
$$

It is instructive to explore the spectral properties of the PML-extended PDE for constant coefficients $m, V, \mathcal{A}$, and $\sigma^{j}=V_{o} / \hbar \geq 0$. Fourier transform of the PML modified PDE gives the dispersion relation

$$
(\hbar \omega-V)^{2}=(c \Pi)^{2} \frac{1}{\left(1+i \frac{V_{o}}{\hbar \omega-V}\right)^{2}}+m^{2},
$$

leading to the fourth-order polynomial in $z=\hbar \omega-V$

$$
z^{4}+2 i V_{o} z^{3}-\left(V_{o}^{2}+(c \Pi)^{2}+m^{2}\right) z^{2}-2 i m^{2} V_{o} z+m^{2} V_{o}^{2}=0
$$

One observes several symmetries: $\left(z, V_{o}\right) \rightarrow\left(z^{*},-V_{o}\right)$ and $\left(z, V_{o} \rightarrow-z,-V_{o}\right)$ leave the polynomial invariant. Thus particle-hole symmetry is preserved. To study how the time-evolution of a wave packet is modified under PML we choose $\Pi$ to be real-valued. For $m=0$, uniform PML amounts to the substitution $V \rightarrow\left(V-i V_{o}\right)$, which is just the IPM for constant $V_{o} \geq 0$. For $m \neq 0$ momentum-dependent modifications arise. For small $\Pi$, the solutions up to order $(c \Pi)^{2}$ are

$$
z_{1,2} \approx \pm m \pm \frac{(c \Pi)^{2}\left(1-\left(V_{o} / m\right)^{2}\right)}{2 m\left(1+\left(V_{o} / m\right)^{2}\right)^{2}}-i V_{o} \frac{(c \Pi)^{2}}{m^{2}\left(1+\left(V_{o} / m\right)^{2}\right)^{2}}
$$

and

$$
z_{3,4} \approx \pm \frac{c|\Pi|}{1+\left(m / V_{o}\right)^{2}}-i V_{o}\left(1-\frac{(c \Pi)^{2}}{m^{2}\left(1+\left(V_{o} / m\right)^{2}\right)^{2}}\right)
$$




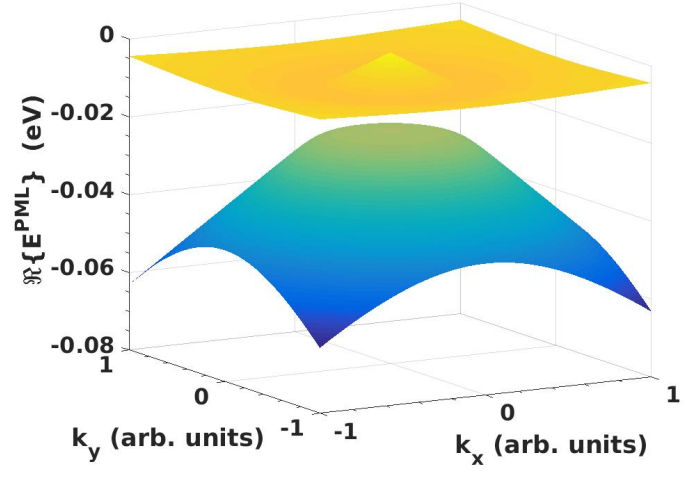

(a)

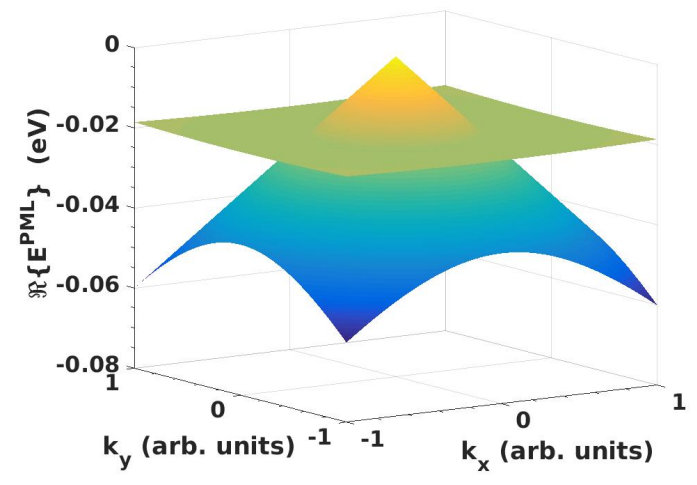

(c)

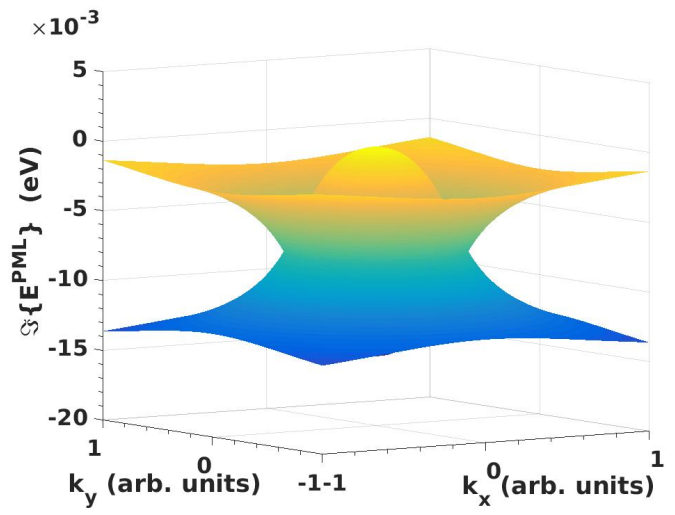

(b)

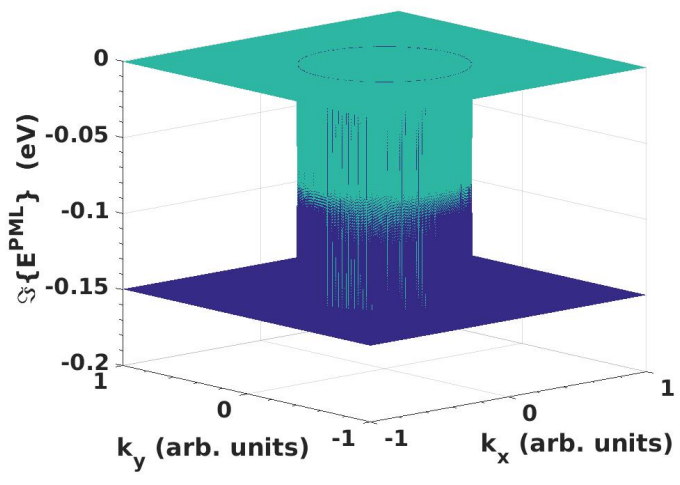

(d)

FIG. 1. (Color Online) Energy dispersion for the PDE under uniform PML for $m=0.02 \mathrm{eV}$ and $\Re\{\hbar \omega-V\} \leq 0, V_{o}=0.015$ $\mathrm{eV}$ : (a) real part, (b) imaginary part; $V_{o}=0.15 \mathrm{eV}$ : (c) real part, (d) imaginary part.

For $V_{o} \rightarrow 0 z_{1,2}$ approaches the non-relativistic limit of a massive particle, whereas $z_{3,4}$ approaches zero. Note the sign dependence of the real part of the kinetic energy on $V_{o} / m$ in Eq. [21), as well as the linear dispersion in Eq. 222. The imaginary part of $z_{1,2}$ and $z_{3,4}$ has the proper (negative) sign needed for exponential damping under time evolution. Imaginary parts of Eq. (21) and (22) add up to $-V_{o}$. The latter holds for all $k$-space. Increasing $V_{o}$ beyond $m$ the real parts of $z_{1}$ and $z_{3}$ and, respectively, $z_{2}$ and $z_{4}$ merge. A numerical example for $z_{2}$ and $z_{4}$ is given in Fig. 1 for $m=0.02 \mathrm{eV}$ and $V_{o}=0.015 \mathrm{eV}$, as well as $V_{o}=0.15 \mathrm{eV}$. Shown is the lower half-cone (negative energy sector) only. It is seen that, for small $k$ and increasing $V_{o}$, the original cone $\left(z_{2}\right)$ is increasingly flattened, while its imaginary part is being reduced to zero from below. Thus, in a PML layer where $V_{o}$ gradually increases from zero to positive values the wave packet will be driven into states of complex energies suffering a combination of damping and slowing down. This behaviour will be found for the FDS under PML as well.

\section{PML FOR THE FINITE-DIFFERENCE SCHEME}

In this section we shall apply the PML substitution to the finite difference scheme (FDS) of Refs. [9, 15]. As the sign of the ratio $v_{g} / k$ is preserved between PDE and this FDS, one may start from the PML extended PDEs derived in the previous chapter. We will consider two PML approaches: The first is based on a FDS where the scalar potential is added to the Hamiltonian explicitly, with the vector potential introduced via a Schwinger substitution. The second approach is based on the free-particle FDS with the entire electromagnetic potential added by a Schwinger substitution. This method preserves gauge invariance (GI) on the grid [15]. 


\section{A. Notation}

For the introduction of PML into the FDS of Hammer et al. we adopt their short-hand notation [9]: any field $f(x, y, t)$ placed on grid point $\left(t=j_{o} \Delta_{t}, x=j_{x} \Delta_{x}, y=j_{y} \Delta_{y}\right)$ is denoted by $f_{j_{x} j_{y}}^{j_{o}}$. Here, $j_{\nu}$ takes integer and halfinteger values. Furthermore, we shall denote the central grid point for an equation by $\left(j_{o}=j_{x}=j_{y}=0\right)$ and relative right-/left shifts (by one half of the respective grid spacing) by \pm . The functional

$$
E_{r_{x}, r_{y}}^{j_{o}}:=\left\|\tilde{u}^{j_{o}+1 / 2}\right\|^{2}+\left\|\tilde{v}^{j_{o}+1}\right\|^{2}+\Re\left[\left(\left(r_{y} \delta_{y}-i r_{x} \delta_{x}\right) \tilde{u}^{j_{o}+1 / 2}, \tilde{v}^{j_{o}+1}\right)\right]\left(=\text { const }=E_{r_{x}, r_{y}}^{0}\right)
$$

is conserved under time propagation within the FDS, periodic or zero boundary conditions, and real-valued mass $m$ and scalar potential $V$. Here we use $r_{x}=c \Delta_{t} / \Delta_{x}$ and $r_{y}=c \Delta_{t} / \Delta_{y}$. Spatial difference operators are written as $\left(\delta_{x} f^{j_{o}}\right)_{j_{x}, j_{y}}=f_{j_{x}+1 / 2, j_{y}}^{j_{o}}-f_{j_{x}-1 / 2, j_{y}}^{j_{o}}$ and $\left(\delta_{y} f^{j_{o}}\right)_{j_{x}, j_{y}}=f_{j_{x}, j_{y}+1 / 2}^{j_{o}}-f_{j_{x}, j_{y}-1 / 2}^{j_{o}}$. The inner product $\left(u^{j_{o}}, v^{j_{o}^{\prime}}\right)_{j^{\prime}}:=$ $\sum_{j} u_{j}^{j_{o}} v_{j+j^{\prime}}^{* j_{o}^{\prime}}=\sum_{j} u_{j-j^{\prime}}^{j_{o}} v_{j}^{* j_{o}^{\prime}}$ is defined on the grid on $l^{2}\left(\mathbb{Z}^{2} ; \mathbb{C}\right) \cdot j^{\prime}$ denotes a primitive displacement vector connecting the spatial sub-lattice of $u$ with that of $v$. We use the notation $\left\|u^{j_{o}}\right\|^{2}:=\left(u^{j_{o}}, u^{j_{o}}\right)$, with the sum over $j$ running over all spatial grid points on time sheet $j_{o}$. The CFL stability condition for the scheme restricts time steps to $\Delta_{t} \leq \Delta_{t}^{C F L}=\Delta /(\sqrt{2} c), \Delta=\Delta_{x}=\Delta_{y}$. For details we refer to the literature [9, 15, 20.

The Schwinger substitution for a spinor component $\psi$ on the grid is defined as follows [9, 10]:

$$
\psi_{j_{x}, j_{y}}^{j_{o}} \rightarrow \tilde{\psi}_{j_{x}, j_{y}}^{j_{o}}=\exp \left\{i a_{j_{x}, j_{y}}^{j_{o}}\right\} \psi_{j_{x}, j_{y}}^{j_{o}},
$$

with the real-valued phase formally given by

$$
a_{j_{x}, j_{y}}^{j_{o}}=\left.\frac{1}{\hbar c_{o}} \int_{\left(x_{o}, y_{o}, c t_{o}\right)}^{(x, y, c t)}\left[d \mathbf{s} \cdot e \mathcal{A}\left(\mathbf{s}, s_{o} / c\right)+\frac{c_{o}}{c} d s_{o} V\left(\mathbf{s}, s_{o} / c\right)\right]\right|_{x=x_{j}, y_{y} y_{j} t=t_{j}} .
$$

The FDS in presence of electromagnetic potentials is obtained by replacing $u, v$ in the free-particle FDS by $\tilde{u}_{j_{x} j_{y}}^{j_{o}}=$ $e^{i a_{j_{x} j_{y}}^{j_{o}}} u_{j_{x} j_{y}}^{j_{o}}, \tilde{v}_{j_{x} j_{y}}^{j_{o}}=e^{i a_{j_{x} j_{y}}^{j_{o}}} v_{j_{x} j_{y}}^{j_{o}}$. Subsequently, the arbitrary local phase is subtracted by multiplication by the phase factor $\exp \left\{-i a_{00}^{0}\right\}$ associated with the central grid position of the specific FDS equation. We define the relative phases $\phi_{00}^{ \pm}=a_{00}^{ \pm}-a_{00}^{0}, \phi_{ \pm 0}^{0}=a_{ \pm 0}^{0}-a_{00}^{0}, \phi_{0 \pm}^{0}=a_{0 \pm}^{0}-a_{00}^{0}$.

\section{B. $\quad V$ in $H$ scheme - introduction of $\mathcal{A}$ via Schwinger substitution}

One starts with Eq. (16) with $V$ in $H$ and $\mathcal{A}=0$. Setting $V=0$ in the phase factor one arrives at the explicit leap-frog scheme

$$
\begin{aligned}
u_{0,0}^{+}-u_{0,0}^{-} & =\frac{\Delta_{t}}{2 i \hbar}(V+m)_{0,0}^{0}\left(u_{0,0}^{+}+u_{0,0}^{-}\right)-r_{y}\left(e^{i \phi_{0,+}^{0}} v_{0,+}^{0}-e^{i \phi_{0,-}^{0}} v_{0,-}^{0}\right. \\
& \left.-\frac{1}{2}\left(\left.\tilde{D}_{y}\right|_{0,0} ^{+}+\left.\tilde{D}_{y}\right|_{0,0} ^{-}\right)\right)-i r_{x}\left(e^{i \phi_{+, 0}^{0}} v_{+, 0}^{0}-e^{i \phi_{-, 0}^{0}} v_{-, 0}^{0}-\frac{1}{2}\left(\left.\tilde{D}_{x}\right|_{0,0} ^{+}+\left.\tilde{D}_{x}\right|_{0,0} ^{-}\right)\right),
\end{aligned}
$$

followed by

$$
\begin{aligned}
v_{0,0}^{+}-v_{0,0}^{-} & =\frac{\Delta_{t}}{2 i \hbar}(V-m)_{0,0}^{0}\left(v_{0,0}^{+}+v_{0,0}^{-}\right)-r_{y}\left(e^{i \phi_{0,+}^{0}} u_{0,+}^{0}-e^{i \phi_{0,-}^{0}} u_{0,-}^{0}\right. \\
& \left.-\frac{1}{2}\left(\left.\tilde{D}_{y}\right|_{0,0} ^{+}+\left.\tilde{D}_{y}\right|_{0,0} ^{-}\right)\right)+i r_{x}\left(e^{i \phi_{+, 0}^{0}} u_{+, 0}^{0}-e^{i \phi_{-, 0}^{0}} u_{-, 0}^{0}-\frac{1}{2}\left(\left.\tilde{D}_{x}\right|_{0,0} ^{+}+\left.\tilde{D}_{x}\right|_{0,0} ^{-}\right)\right) .
\end{aligned}
$$

The grid values $\left.\tilde{D}_{x}\right|_{0,0} ^{0}$ and $\left.\tilde{D}_{y}\right|_{0,0} ^{0}$ needed in Eqs. 26) and 27) must yet be defined. In the spirit of the underlying leap-frog scheme for the mass and potential term, we apply the time-symmetric average

$$
\left.\tilde{D}_{x}\right|_{0,0} ^{0} \equiv \frac{1}{2}\left(\left.\tilde{D}_{x}\right|_{0,0} ^{+}+\left.\tilde{D}_{x}\right|_{0,0} ^{-}\right),\left.\tilde{D}_{y}\right|_{0,0} ^{0}=\frac{1}{2}\left(\left.\tilde{D}_{y}\right|_{0,0} ^{+}+\left.\tilde{D}_{y}\right|_{0,0} ^{-}\right) .
$$

The PML terms $\left.D_{x}\right|_{j_{x}, j_{y}} ^{j_{o}}$ and $\left.D_{y}\right|_{j_{x}, j_{y}} ^{j_{o}}$, respectively, may be computed based on the PDEs 13. and (14). This approach leads one to

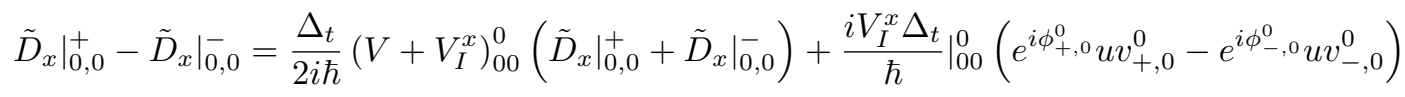


and

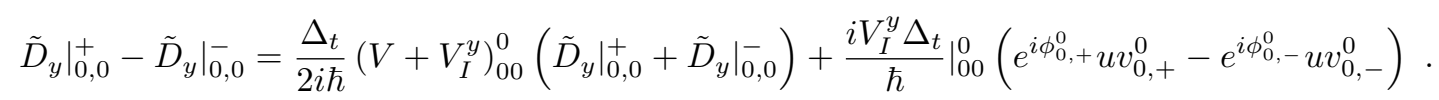

Here, $u v_{j_{x}, j_{y}}^{j_{o}}$ denotes $u_{j_{x}, j_{y}}^{j_{o}}$ for a $u$-grid position, and $v_{j_{x}, j_{y}}^{j_{o}}$ for a $v$-grid position. Note that $V$ enters Eqs. 29 and (30) via $\mathbf{v}_{j}(\mathbf{k})=\frac{c^{2} \hbar k_{j}}{\hbar \omega-V}$ in the PML substitution Eq. (7). For future reference, we term this FDS the VinHCN scheme. Alternatively, starting from Eqs. (17) and (18) and using the approximation

$$
\int_{-\Delta_{t} / 2}^{\Delta_{t} / 2} f_{j_{x} j_{y}}^{t} d t=\Delta_{t} f_{j_{x} j_{y}}^{0}+\mathbb{O}\left(\Delta_{t}^{3}\right)
$$

one obtains the scheme

$$
\left.\tilde{D}_{x}\right|_{0,0} ^{+}=\left.e^{-i \frac{\Delta_{t}}{\hbar}\left(V+V_{I}^{x}\right)_{00}^{0}} \tilde{D}_{x}\right|_{0,0} ^{-}+\left.\left(1-e^{-i \frac{\Delta_{t}}{\hbar}\left(V+V_{I}^{x}\right)_{00}^{0}}\right) \frac{V_{I}^{x}}{V+V_{I}^{x}}\right|_{00} ^{0}\left(e^{\left.i \phi_{+, 0}^{0} u v_{+, 0}^{0}-e^{i \phi_{-, 0}^{0}} u v_{-, 0}^{0}\right)}\right.
$$

and

$$
\left.\tilde{D}_{y}\right|_{0,0} ^{+}=\left.e^{-i \frac{\Delta_{t}}{\hbar}\left(V+V_{I}^{y}\right)_{00}^{0}} \tilde{D}_{y}\right|_{0,0} ^{-}+\left.\left(1-e^{-i \frac{\Delta_{t}}{\hbar}\left(V+V_{I}^{y}\right)_{00}^{0}}\right) \frac{V_{I}^{y}}{V+V_{I}^{y}}\right|_{00} ^{0}\left(e^{\left.i \phi_{0,+}^{0} u v_{0,+}^{0}-e^{i \phi_{0,-}^{0}} u v_{0,-}^{0}\right)} .\right.
$$

In the end, both versions are $\mathbb{O}\left(\Delta_{t}^{2}\right)$ accurate via Eq. 31 . We term the latter the VinHEXP scheme.

Pinaud apparently proposed a slightly different $\mathbb{O}\left(\Delta_{t}^{2}\right)$ accurate scheme [11]. Studying the case of zero electromagnetic potentials, he defines $\left.D_{x}\right|_{0,0} ^{0}$ and $\left.D_{y}\right|_{0,0} ^{0}$ via a time-asymmetric (retarded) time average:

$$
\begin{aligned}
& \left.\left.D_{x}\right|_{0,0} ^{0} \approx e^{-\sigma_{00} \Delta_{t}} D_{x}\right|_{0,0} ^{--}+\left(1-e^{-\sigma_{00} \Delta_{t}}\right) \frac{1}{2}\left(u v_{+, 0}^{0}-u v_{-, 0}^{0}+u v_{+, 0}^{--}-u v_{-, 0}^{--}+\right), \\
& \left.\left.D_{y}\right|_{0,0} ^{0} \approx e^{-\sigma_{00} \Delta_{t}} D_{y}\right|_{0,0} ^{--}+\left(1-e^{-\sigma_{00} \Delta_{t}}\right) \frac{1}{2}\left(u v_{0,+}^{0}-u v_{0,-}^{0}+u v_{0,+}^{--}-u v_{0,-}^{-}\right) .
\end{aligned}
$$

where -- denotes the relative time shift by $-c \Delta_{t}$. This scheme computes the spatial derivatives as a time average at time - i.e., the respective initial time for Eqs. 26] and (27), using

$$
\int_{-\Delta_{t}}^{0} f_{j_{x} j_{y}}^{t} d t=\frac{\Delta_{t}}{2}\left(f_{j_{x} j_{y}}^{0}+f_{j_{x} j_{y}}^{--}\right)+\mathbb{O}\left(\Delta_{t}^{3}\right)
$$

\section{GI schemes - introduction of $V$ and $\mathcal{A}$ via Schwinger substitution}

Setting the electromagnetic potentials $V$ and $\mathcal{A}$ equal to zero in Eq. 16 and putting them into the Schwinger phase factor one obtains

$$
\begin{aligned}
e^{i \phi_{0,0}^{+}} u_{0,0}^{+}-e^{i \phi_{0,0}^{-}} u_{0,0}^{-} & =\left.\frac{\Delta_{t}}{2 i \hbar} m\right|_{0,0} ^{0}\left(e^{i \phi_{0,0}^{+}} u_{0,0}^{+}+e^{i \phi_{0,0}^{-}} u_{0,0}^{-}\right)-r_{y}\left(e^{i \phi_{0,+}^{0} v_{0,+}^{0}}-e^{i \phi_{0,-}^{0}} v_{0,-}^{0}\right. \\
& \left.-\frac{1}{2}\left(\left.\tilde{D}_{y}\right|_{0,0} ^{+}+\left.\tilde{D}_{y}\right|_{0,0} ^{-}\right)\right)-i r_{x}\left(e^{i \phi_{+, 0}^{0}} v_{+, 0}^{0}-e^{i \phi_{-, 0}^{0} v_{-, 0}^{0}}-\frac{1}{2}\left(\left.\tilde{D}_{x}\right|_{0,0} ^{+}+\left.\tilde{D}_{x}\right|_{0,0} ^{-}\right)\right)
\end{aligned}
$$

followed by

$$
\begin{aligned}
e^{i \phi_{0,0}^{+}} v_{0,0}^{+}-e^{i \phi_{0,0}^{-}} v_{0,0}^{-} & =-\left.\frac{\Delta_{t}}{2 i \hbar} m\right|_{0,0} ^{0}\left(e^{i \phi_{0,0}^{+}} v_{0,0}^{+}+e^{i \phi_{0,0}^{-}} v_{0,0}^{-}\right)-r_{y}\left(e^{i \phi_{0,+}^{0} u_{0,+}^{0}-e^{i \phi_{0,-}^{0}} u_{0,-}^{0}}\right. \\
& \left.-\frac{1}{2}\left(\left.\tilde{D}_{y}\right|_{0,0} ^{+}+\left.\tilde{D}_{y}\right|_{0,0} ^{-}\right)\right)+i r_{x}\left(e^{i \phi_{+, 0}^{0}} u_{+, 0}^{0}-e^{i \phi_{-, 0}^{0}} u_{-, 0}^{0}-\frac{1}{2}\left(\left.\tilde{D}_{x}\right|_{0,0} ^{+}+\left.\tilde{D}_{x}\right|_{0,0} ^{-}\right)\right) .
\end{aligned}
$$

The PML functions may be computed from a discretisation of Eq. (15),

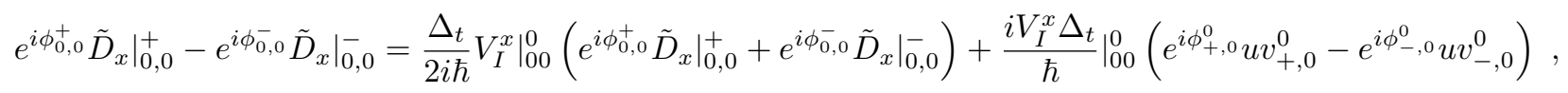

and

$$
\left.e^{i \phi_{0,0}^{+}} \tilde{D}_{y}\right|_{0,0} ^{+}-\left.e^{i \phi_{0,0}^{-}} \tilde{D}_{y}\right|_{0,0} ^{-}=\left.\frac{\Delta_{t}}{2 i \hbar} V_{I}^{y}\right|_{00} ^{0}\left(\left.e^{i \phi_{0,0}^{+}} \tilde{D}_{y}\right|_{0,0} ^{+}+\left.e^{i \phi_{0,0}^{-}} \tilde{D}_{y}\right|_{0,0} ^{-}\right)+\left.\frac{i V_{I}^{y} \Delta_{t}}{\hbar}\right|_{00} ^{0}\left(e^{\left.i \phi_{0,+}^{0} u v_{0,+}^{0}-e^{i \phi_{0,-}^{0}} u v_{0,-}^{0}\right)}\right.
$$

or the integral equation leading to Eqs. 32 with $V$ moved into the phase factors. For future reference, we term these two versions the GICN, respectively, GIEXP scheme. 


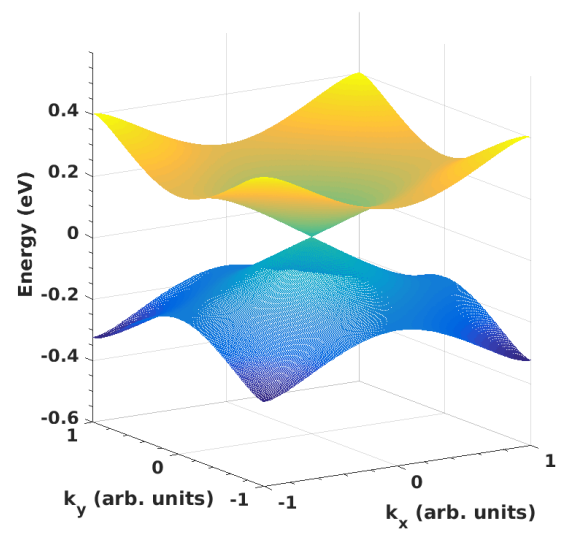

(a)

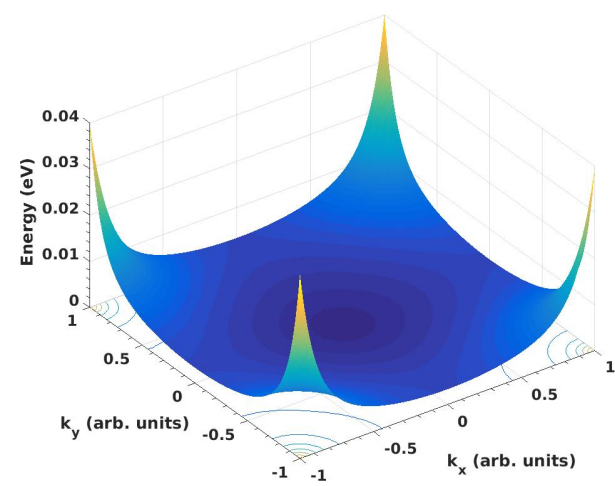

(c)

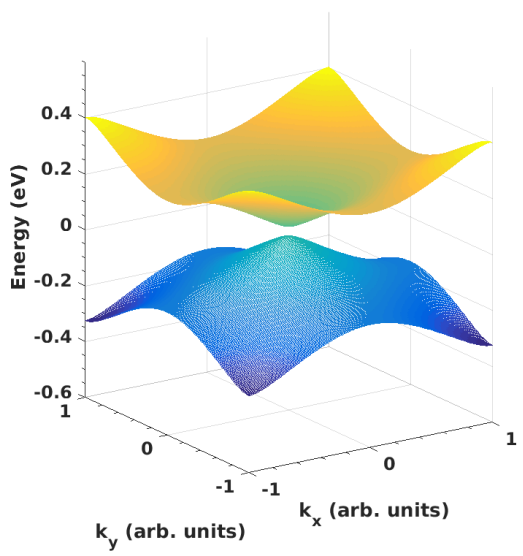

(b)

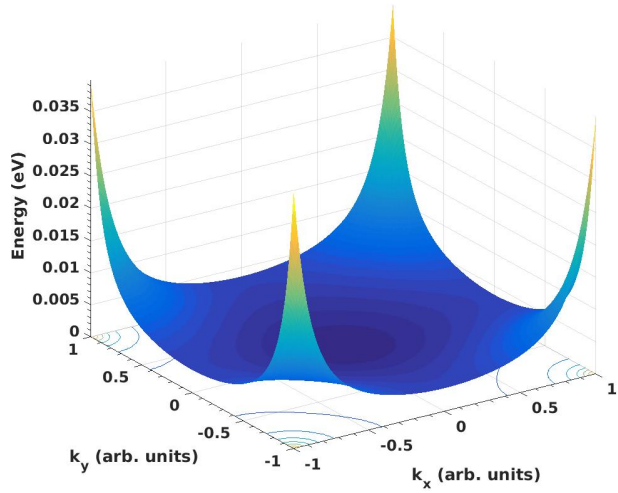

(d)

FIG. 2. (Color Online) Energy dispersion $E=\hbar \omega$ of the VinH scheme for $V=0.04 \mathrm{eV}$ : (a) $m=0 \mathrm{eV}$; (b) $m=0.02 \mathrm{eV}$; energy difference to the GI scheme, $\left.\hbar \omega\right|_{V=0}+V-\left.\hbar \omega\right|_{V}$, for (c) $m=0 \mathrm{eV}$, (d) $m=0.02 \mathrm{eV}$.

\section{SPECTRAL PROPERTIES UNDER CONSTANT ELECTROMAGNETIC POTENTIALS}

\section{A. Introduction of the scalar potential}

First we investigate how the introduction of the scalar potential influences the spectral properties. Putting the term $\Delta_{t} V\left(u v_{00}^{+}+u v_{00}^{-}\right) /(2 i \hbar)$ into the FDS, as in Eqs. (26) and (27), preserves all the stability and convergence features of the scheme. However, it does not provide a strictly GI introduction of $V$ into the FDS. In particular, adding a constant scalar potential modifies the energy dispersion and thus the group velocity. This can be seen from the energy dispersion obtained via Fourier analysis from the FDS with the $V$ term $(\mathcal{A}=0)$ in place. For $\mathcal{A} \neq 0$ but constant one simply has to replace $\hbar \mathbf{k}$ by $\boldsymbol{\Pi}$. One finds

$$
\begin{gathered}
\hbar \omega= \pm \frac{2 \hbar}{\Delta_{t}} \arcsin \left[B \pm \sqrt{B^{2}-C}\right]^{1 / 2} \\
B=\frac{X Y+2 Z^{2}}{Y^{2}+4 Z^{2}}, C=\frac{X^{2}}{Y^{2}+4 Z^{2}}, X=+M^{2}-Z^{2}, Y=1+M^{2}-Z^{2},
\end{gathered}
$$




$$
K_{o}^{2}=\left(r_{x}\right)^{2} \sin ^{2}\left\{\frac{\Delta_{x} k_{x}}{2}\right\}+\left(r_{y}\right)^{2} \sin ^{2}\left\{\frac{\Delta_{y} k_{y}}{2}\right\}, M=\frac{m \Delta_{t}}{2 \hbar}, Z=\frac{V \Delta_{t}}{2 \hbar} .
$$

The four solutions for $V \neq 0$ correspond to $V$ and $-V$ and can be associated with one or the other by inspection of the sign of

$$
\frac{\left(X-Y \sin ^{2}\left(\alpha_{\omega}\right)\right)}{\sin \left(\alpha_{\omega}\right) \cos \left(\alpha_{\omega}\right)}\left(=\frac{V \Delta_{t}}{\hbar}\right), \alpha_{\omega}=\frac{\omega \Delta_{t}}{2}
$$

For $V=0, B^{2}-C=0$ and one ends up with the single-cone solution (one pair of solutions)

$$
\hbar \omega= \pm \frac{2 \hbar}{\Delta_{t}} \arcsin \left[\frac{K_{o}^{2}+M^{2}}{1+M^{2}}\right]^{1 / 2} .
$$

There is no fermion doubling. However, $\left.\hbar \omega\right|_{V} \neq\left.\hbar \omega\right|_{V=0}+V$.

When introducing $V$ via the substitution $\psi(t) \rightarrow \tilde{\psi}=\exp \{i V t / \hbar\} \psi(t)$, the variation $\psi_{00}^{ \pm}=\exp \left\{\mp i \omega \Delta_{t} / 2\right\} \psi_{00}^{0}$ transforms into $\left.\tilde{\psi}_{00}^{ \pm}=\exp \left\{ \pm i(V / \hbar-\omega) \Delta_{t}\right) / 2\right\} \psi_{00}^{0}$. Likewise, the introduction of $A_{x}$ and $A_{y}$ leads to a shift of, respectively, $k_{x}$ and $k_{y}$. The spectral properties of the FDS Eq. (37) and (38) under periodic or zero boundary conditions for $D_{x}=D_{y}=0, V, A_{x}$ and $A_{y}$ constan are obtained from the $V=A_{x}=A_{y}=0$ case, Eq. 445), by the substitution $\omega \rightarrow \omega-V / \hbar, k_{x} \rightarrow \Pi_{x}, k_{y} \rightarrow \Pi_{y}$. As a result, the single-cone is shifted rigidly along $\omega$, respectively, $k_{x}$ and/or $k_{y}$, as required under gauge invariance. In particular, the group velocity of this scheme is GI.

For a numerical comparison of the dispersion relation obtained from the two approaches, we consider a rectangular domain $L_{x} \times L_{y}$ and $N_{x} \times N_{y}$ grid points for storing the spinor components $u v_{j_{x}, j_{y}}^{j_{t}}$ at given time $j_{t}=j_{o}-1 / 2, j_{o}$. Note that two adjacent time sheets are needed within the leap-frog scheme. We set $L_{x}=L_{y}=1 \mathrm{~nm}, N_{x}=N_{y}=400$, $c=6.2 \times 10^{5} \mathrm{~m} / \mathrm{s}$. Figs. 2 (a) and (b) give the single-cone energy dispersion $\hbar \omega(V)$ from Eq. 41) for $V=0.04$ $\mathrm{eV}$ and $m=0 \mathrm{eV}$ and, respectively, $m=0.02 \mathrm{eV}$. Here $k$ is measured in units of $\pi / \Delta, \Delta=\Delta_{x}=\Delta_{y}$. We use the maximum time step under CFL, i.e., $c \Delta_{t}=\Delta / \sqrt{2}$. While reduced in absolute number, the sign of the ratio $v_{g}(k) / k$ in the FDS is preserved when compared to the exact ratio from the PDE. Just at the four k-points $( \pm \pi / \Delta, 0)$ and $(0, \pm \pi / \Delta) v_{g}$ approaches zero. Figs. 2 (c) and (d) give the energy difference $\left.\hbar \omega\right|_{V=0}+V-\left.\hbar \omega\right|_{V}$ between the upper bands. The maximum difference occurs at the diagonal corners of the zone boundary and is about 10 percent of $V$, with the GI scheme giving a slightly better approximation to the PDE dispersion (larger values for both $\hbar \omega$ and $v_{g}$ ). For PML these differences are insignificant in that the sign of $v_{g}$ remains unchanged. Both schemes can be subjected to the PML substitution derived from the PDE.

\section{B. Spectral properties of the PML augmented schemes}

First we explore the spectral changes to the FDS introduced by the PML substitution, setting $\gamma^{j}\left(x_{j}\right)=\sigma x_{j}, x_{j}=$ $x, y$, with $\sigma=i V_{I} / \hbar \geq 0, V$, and $\mathcal{A}$ constant. Thus, the PML substitution Eq. (7) reads

$$
\Pi_{x}(k) x \rightarrow \Pi_{x}(k)\left(1+i \sigma \frac{v_{x}\left(\Pi_{x}, \Pi_{y}\right)}{\Pi_{x}}\right) x=\Pi_{x}(k)\left(1+i \sigma \frac{c^{2}}{\hbar \omega-V}\right) x, \sigma \geq 0 .
$$

This allows two equivalent interpretations: (i) $x$ is replaced by $x^{\prime}=\left(1+i \sigma \frac{c^{2}}{\hbar \omega-V}\right) x$ (PML substitution), or (ii) $\Pi_{x}(k) \rightarrow \Pi_{x}^{\prime}(k)=\Pi_{x}(k)\left(1+i \sigma \frac{c^{2}}{\hbar \omega-V}\right)$.

Within scheme Eqs. 228), 29, and (30), the modifications to Eq. 45) are obtained by the substitution

$$
K_{o}^{2} \rightarrow K^{2}=K_{o}^{2}\left(1+\frac{\sigma \Delta_{t} \cos \left(\alpha_{\omega}\right)}{2 i \sin \left(\alpha_{\omega}\right)-\sigma \Delta_{t} \cos \left(\alpha_{\omega}\right)}\right)^{2}=K_{o}^{2}\left(\frac{1}{1+i \sigma \frac{\Delta_{t}}{2} \cot \left(\alpha_{\omega}\right)}\right)^{2} .
$$

For the PML scheme based on Eqs. (28), 232), and (33) it is

$$
K_{o}^{2} \rightarrow K^{2}=K_{o}^{2}\left(1-\frac{\left(1-e^{-\sigma \Delta_{t}}\right) \cos \left(\alpha_{\omega}\right)}{e^{-i \alpha_{\omega}}-e^{-\sigma \Delta_{t}} e^{i \alpha_{\omega}}}\right)^{2}=K_{o}^{2}\left(\frac{1}{1+i \tanh \left(\sigma \Delta_{t} / 2\right) \cot \left(\alpha_{\omega}\right)}\right)^{2} .
$$

Both Eqs. (47) and 48) lead back to Eq. (19) in the limit $\Delta_{t} \rightarrow 0$. We note that the time-averaging Eq. (36) adopted by Pinaud too leads to Eq. 48. 


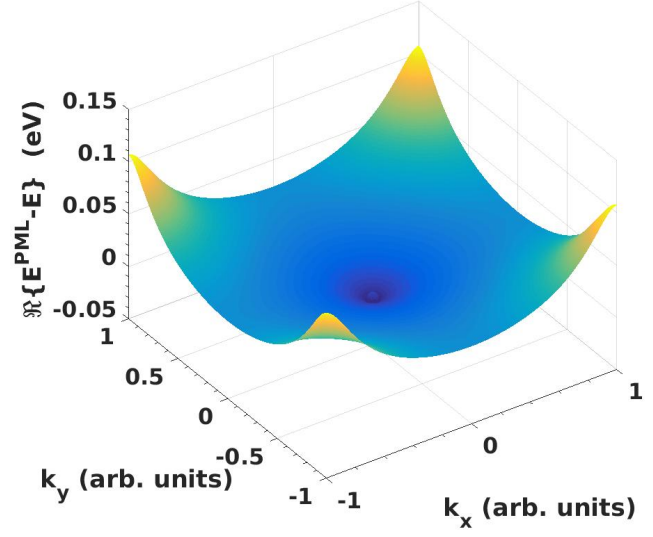

(a)

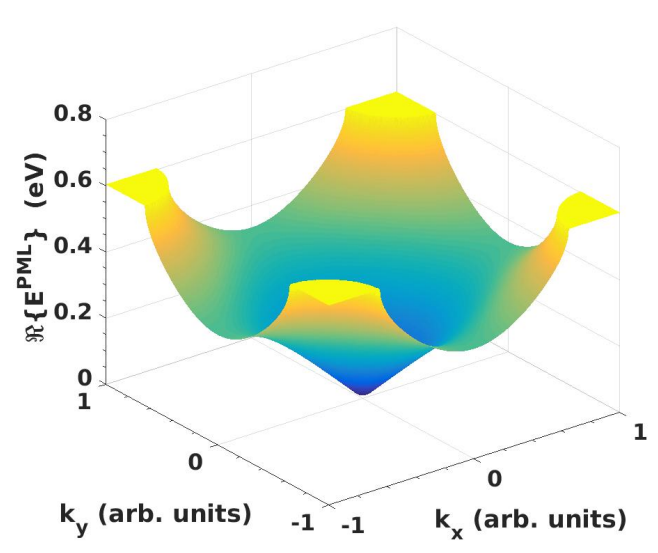

(c)

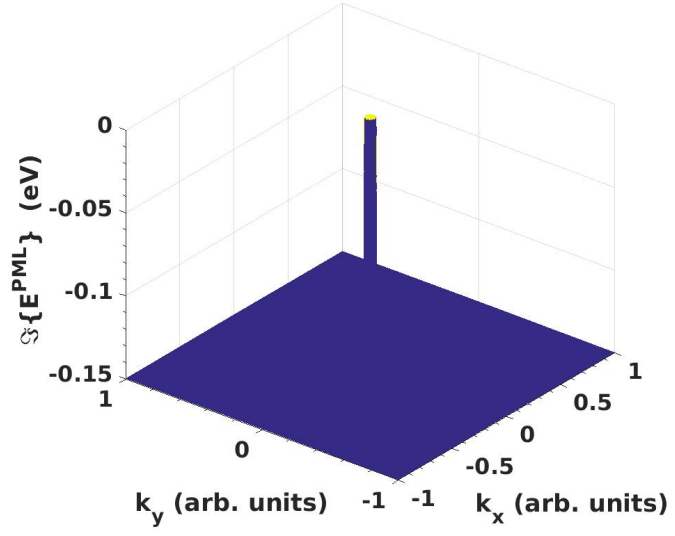

(b)

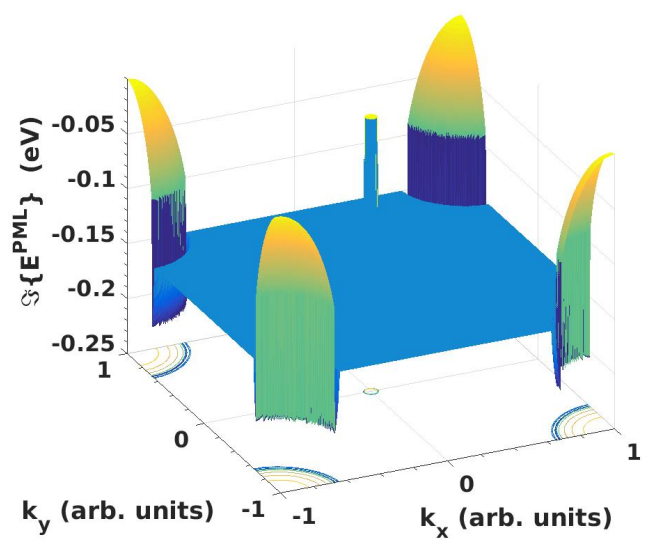

(d)

FIG. 3. (Color Online) Energy dispersion for the GIEXP scheme under uniform PML for $m=0.02 \mathrm{eV}$ and $\Re\{\hbar \omega-V\} \geq 0$, $V_{o}=0.15 \mathrm{eV}$ : PML-induced energy difference for $\Delta_{t}$ from Eq. 55): (a) real part, (b) imaginary part; energy dispersion with $\mathrm{PML}$ and $\Delta_{t}=\Delta_{t}^{C F L}$ : (c) real part, (d) imaginary part.

We start with $M=0$. Replacing $K_{o}^{2}$ by $K^{2}$ in Eq. 45, one obtains

$$
\sin \left(\alpha_{\omega}\right)+i \alpha \cos \left(\alpha_{\omega}\right)= \pm K_{o}
$$

with $\alpha=\sigma \Delta_{t} / 2$ and $\tanh \left(\sigma \Delta_{t} / 2\right)$, respectively, for Eq. 477) and 48). This may be recast into

$$
\sin \left(\alpha_{\omega}+i b\right)= \pm K_{o} \frac{1}{\sqrt{1-\alpha^{2}}}, b=\operatorname{arcCosh}\left(\frac{1}{\sqrt{1-\alpha^{2}}}\right)
$$

or

$$
\alpha_{\omega}= \pm \arcsin \left(K_{o} \frac{1}{\sqrt{1-\alpha^{2}}}\right)-i \operatorname{arcCosh}\left(\frac{1}{\sqrt{1-\alpha^{2}}}\right) .
$$

For the exponential scheme one obtains

$$
\alpha_{\omega}= \pm \arcsin \left(K_{o} \cosh \left(\sigma \Delta_{t} / 2\right)\right)-i \sigma \Delta_{t} / 2 .
$$

Therefore

$$
r_{x}^{2}+r_{y}^{2} \leq 1-\alpha^{2}, 0 \leq \alpha<1
$$


ensures the negative imaginary part needed for exponential damping under forward-in-time evolution and real-valued $K_{o}$. For $r_{x}=r_{y}=r$, one obtains the condition

$$
r \leq \frac{1}{\sqrt{2}} \frac{1}{\sqrt{1+\frac{1}{2}\left(\frac{\Delta \sigma}{2 c}\right)^{2}}}
$$

using Eq. 47), and

$$
r \cosh \left(\frac{\Delta \sigma}{2 c} r\right) \leq \frac{1}{\sqrt{2}}
$$

for Eq. (48). Owing to the structure of the argument on the rhs. of Eq. (45), the same conditions hold for $M \neq 0$. They replace the CFL conditions for the underlying FDS.

Respecting the new CFL conditions, the PML induced spectral changes within the FDS are qualitatively similar to the ones for the PDE discussed in Sect. II] A numerical example for the deformation of the upper half of a Dirac cone is shown in Fig. 3. We use $M=0.02 \mathrm{eV}$ and $V_{o}=0.15 \mathrm{eV}$ and $600 \times 600$ grid points for an area of $900 \mathrm{~nm}$ $\times 900$ nm. Fig. 3 (a) and (b) show the PML-induced changes of the cone relative to the FDS dispersion in absence of PML. The time step which enters the dispersion is set to the maximum value meeting condition (55). One observes a widening of the cone for small $k$, but not nearly as pronounced as for the PDE, and a steepening for large $k$. The imaginary part is near constant at value $V_{o}$, except for small $k$ where it tends to zero from below. Fig. 3 (c) and (d) show the effects on the spectrum when using $\Delta_{t}=\Delta_{t}^{C F L}=\Delta /(\sqrt{2} c)$. At the corners of the Brillouin zone the cone flattens, just as it does in absence of PML when $\Delta_{t}$ exceeds $\Delta_{t}^{C F L}$. The imaginary part now approaches zero from below at these corners as well.

\section{NUMERICAL TESTS}

The level of performance of a PML scheme will be determined by monitoring the time evolution of the functional Eq. 23. Also shown will be the lattice trace which is comprised of the first two terms in $E_{r_{x}, r_{y}}^{j_{o}}$.

For a numerical simulation we need to select PML damping functions $V_{I}^{x}(x)$ and $V_{I}^{y}(y)$. We choose

$$
V_{I}^{x}(x)=i V_{o}\left(\frac{1}{1+e^{x / W}}+\frac{1}{1+e^{\left(L_{x}-x\right) / W}}\right), 0 \leq x \leq L_{x},
$$

and

$$
V_{I}^{y}(y)=i V_{o}\left(\frac{1}{1+e^{y / W}}+\frac{1}{1+e^{\left(L_{y}-y\right) / W}}\right), 0 \leq y \leq L_{y} .
$$

Layer width $W$ and damping potential $V_{o}$ may be tuned for optimal performance. We measure $W$ in units of the $u v$ grid spacing $\Delta / 2$.

The efficiency of the PML schemes is compared to the one for the IPM. The latter consists in the substitution $V \rightarrow V+V_{I}$ in the FDS. Here, $V$ denotes the physical scalar potential energy, and $V_{I}$ is the imaginary potential contribution. This simple method works quite well for the Dirac equation and does not affect the original CFL condition. In general, the sum $V_{I}(x, y)=V_{I}^{x}(x)+V_{I}^{y}(y)$ will serve for our imaginary potential to compare with a PML simulation.

\section{A. Uniform PML layer}

In this section we compare the four schemes VinHEXP, VinHCN, GIEXP, and GICN under uniform PML. We choose a $600 \times 600$ grid for $u v$ on a $1 \mathrm{~nm} \times 1 \mathrm{~nm}$ sheet under uniform potentials $V=0.05 \mathrm{eV}, V_{o}=0.08 \mathrm{eV}$, respectively, $V_{o}=0.8 \mathrm{eV}$. A Dirac fermion of mass $m=0.02 \mathrm{eV}$ starts in a Gaussian-wave-packet state placed at the centre of the simulation region. It sets out with a kinetic energy of $E_{x}=c \hbar k_{x}=0.15 \mathrm{eV}, k_{y}=0$. The width of the wave packet is $50 \Delta$. Simulation time is $600 \Delta_{t}^{C F L}=2.28 \times 10^{-12} \mathrm{~s}$. The actual number of time steps is 615 for $V_{o}=0.08$ $\mathrm{eV}$; for $V_{o}=0.8 \mathrm{eV}$ it is 1120 and 1510, respectively, for the $E X P$ and $C N$ integration schemes. Results for the GI schemes are shown in Fig. 4 blue (solid) lines give the time evolution of functional Eq. (23), red (dashed) lines give the lattice trace versus time. Fig. 4(a) and 4(b) give the results for scheme GIEXP for, respectively, $V_{o}=0.08$ 


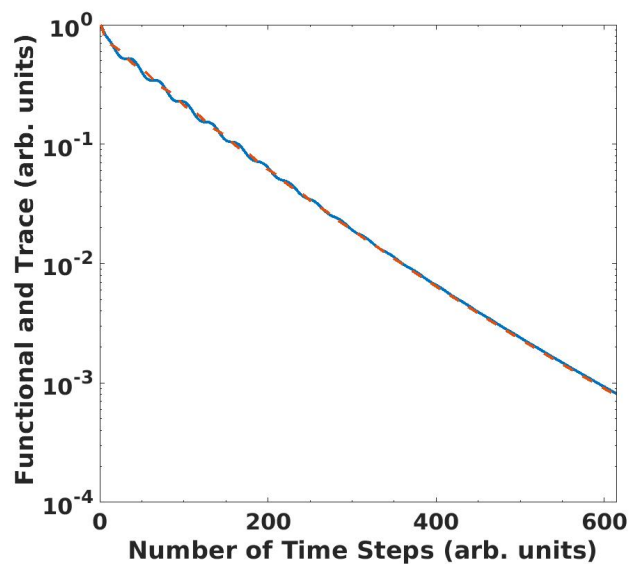

(a)

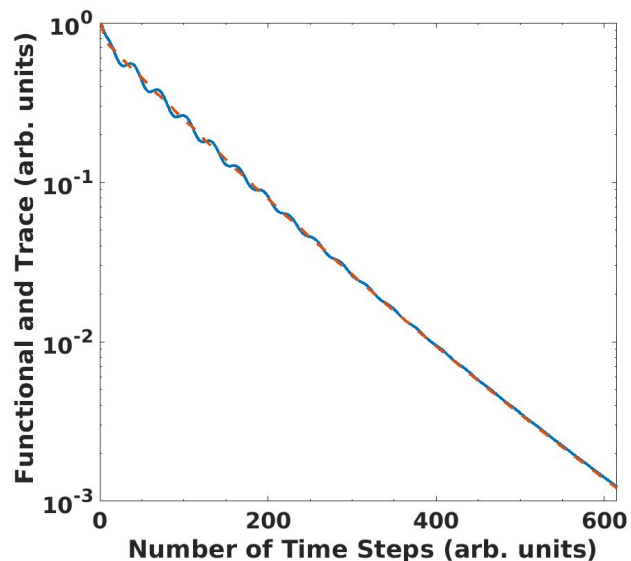

(c)

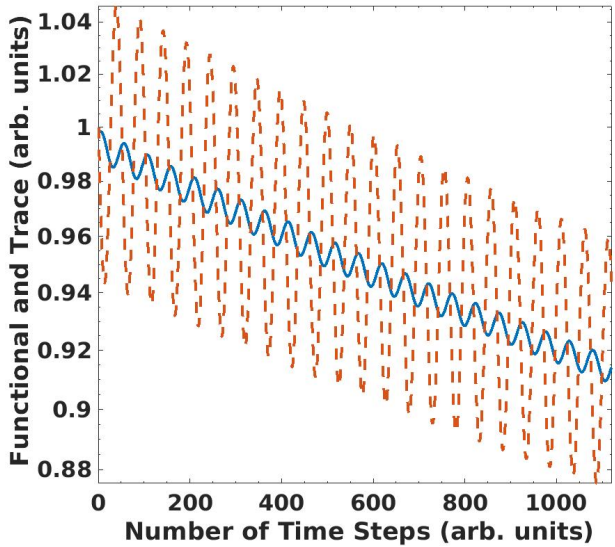

(b)

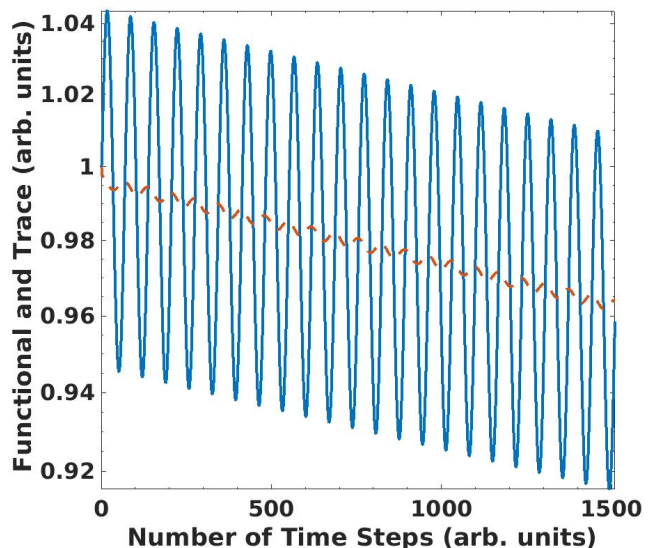

(d)

FIG. 4. (Color Online) Time-evolution of the functional Eq. 23) (solid blue lines) and lattice trace (dashed red lines) under uniform PML: (a) scheme GIEX for $V_{o}=0.08 \mathrm{eV}$, (b) scheme GIEX for $V_{o}=0.8 \mathrm{eV}$, (c) scheme GICN for $V_{o}=0.08 \mathrm{eV}$, (b) scheme GICN for $V_{o}=0.8 \mathrm{eV}$. Note the logarithmic scale along the y-axis.

$\mathrm{eV}$ and $V_{o}=0.8 \mathrm{eV}$. Fig. 4 (c) and 4(d) give the results obtained within scheme GICN. Note the logarithmic scale used for the $y$-axes. One observes a somewhat better performance of the GIEXP scheme over the GICN scheme, in particular, since the former allows the use of shorter time steps. Moreover, damping for $V_{o}=0.08 \mathrm{eV}$ is stronger than for $V_{o}=0.8 \mathrm{eV}$. This is counter-intuitive from the stand point of the IPM, however, well in agreement with PML induced spectral changes in form of a flattening of the energy dispersion in the small-k region, as seen in Figs. 33(a) and (b), and 1 Small to moderate $V_{o}$ leads to damping, moderate to large $V_{o}$ leads to trapping. The effective group velocity approaches zero. Stronger damping is observed for higher $\mathbf{k}$ and/or zero mass $m$. In the case of uniform $V_{I}$, IPM outperforms PML. This changes in favour of PML when absorbing boundary layers are considered.

\section{B. Picture frame PML}

Now we compare the four approaches using the damping functions Eqs. (56) and (57). This corresponds to a picture frame boundary region $V_{I}(x, y)=V_{I}^{x}(x)+V_{I}^{y}(y)$. We investigate results for $W=6(\Delta / 2)$ and $V_{o}=0.5 \mathrm{eV}$. The initial wave packet is given a kinetic energy $E_{x}=0.015 \mathrm{eV}, E_{y}=0$. It is heading towards the right boundary of the simulation region. The simulation is carried out from time zero to $t=1000 \Delta_{t}^{C F L}$. All other parameters are as in the previous section. Our findings are collected in Fig. 6. Shown is the log of functional Eq. (23) versus time step. One observes that all four PML schemes lead to practically identical results, however, the CN-based schemes accomplish the task within 1756 time steps to safely stay within stability limits, whereas the two EXP-based schemes 


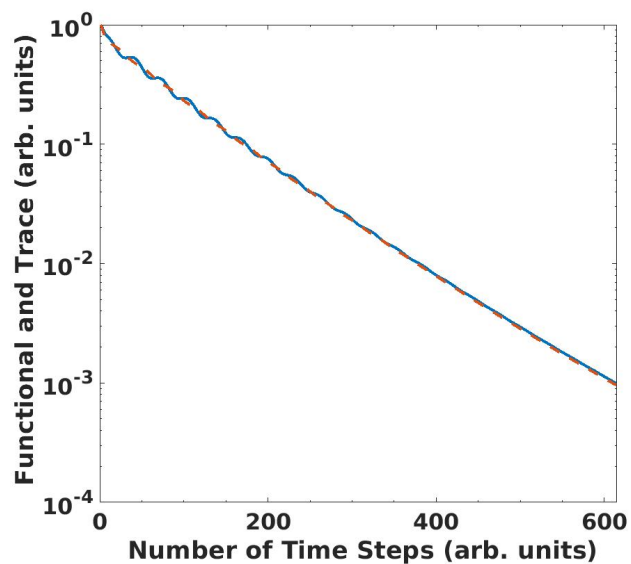

(a)

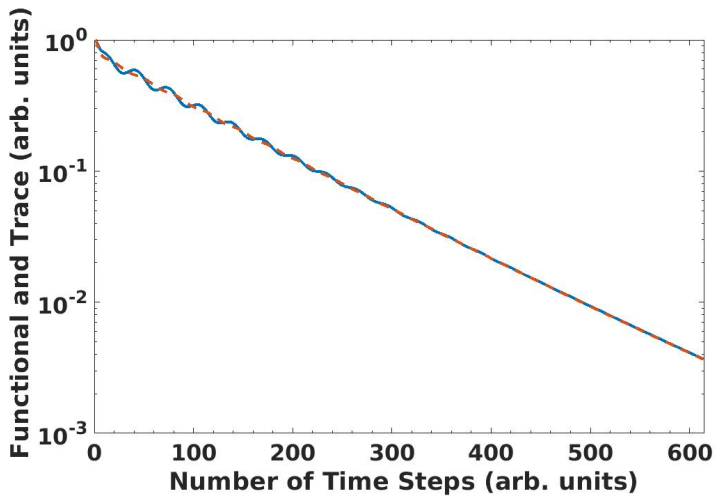

(c)

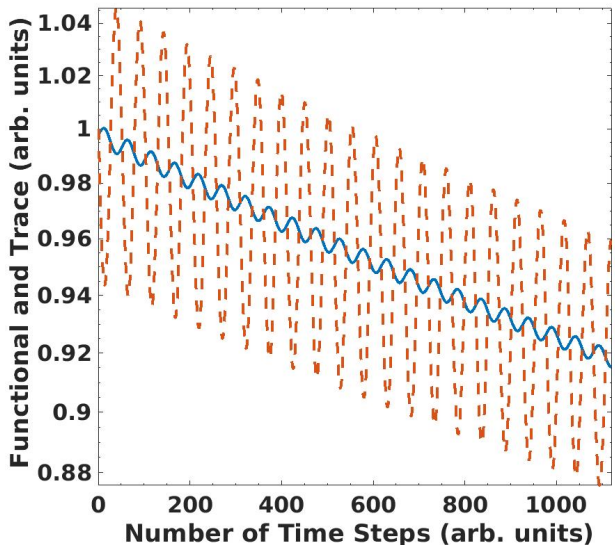

(b)

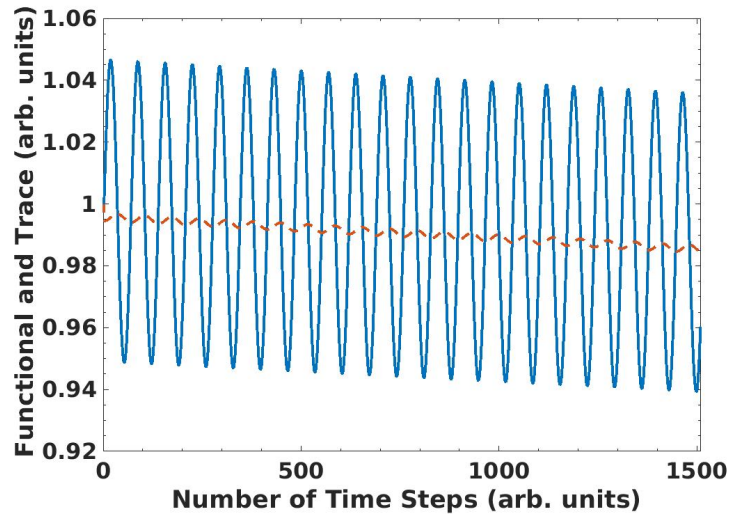

(d)

FIG. 5. (Color Online) Time-evolution of the functional Eq. 23) (solid blue lines) and lattice trace (dashed red lines) under uniform PML: (a) scheme VinHEX for $V_{o}=0.08 \mathrm{eV}$, (b) scheme VinHEX for $V_{o}=0.8 \mathrm{eV}$, (c) scheme VinHCN for $V_{o}=0.08$ $\mathrm{eV}$, (b) scheme VinHCN for $V_{o}=0.8 \mathrm{eV}$. Note the logarithmic scale along the y-axis.

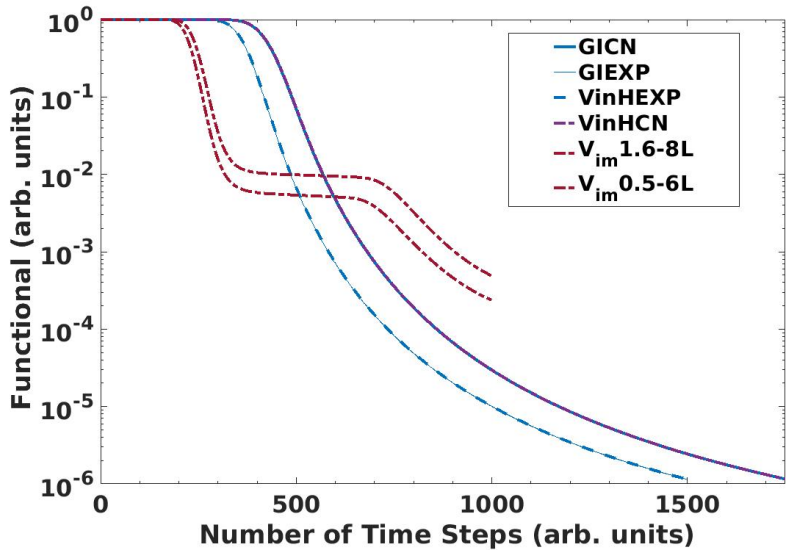

FIG. 6. (Color Online) Comparison of PML scheme performance for free-particle propagation $(m=0.02 \mathrm{eV})$ on a domain of constant scalar potential $V=0.05 \mathrm{eV}$ and picture frame PML Eqs. (56) and (57) for $W=6(\Delta / 2), V=0.05 \mathrm{eV}$ and $V_{o}=0.5$ $\mathrm{eV}$. Results within IPM (red dot-dashed lines labeled $V_{\mathrm{im}}$ ) are given for comparison. 


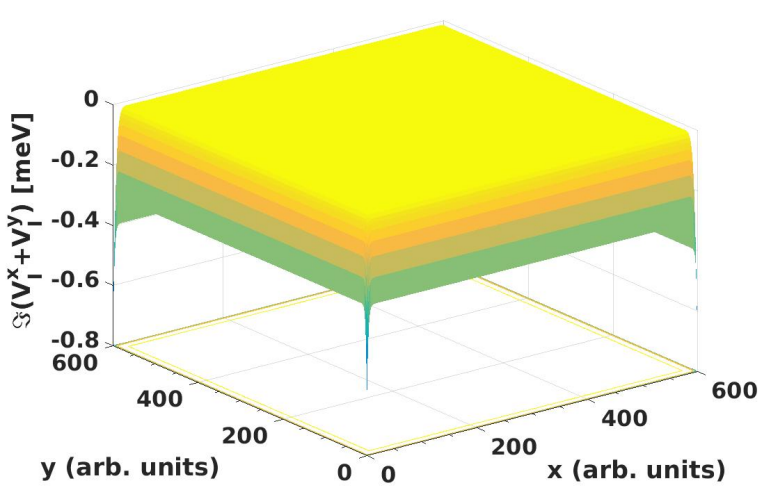

(a)

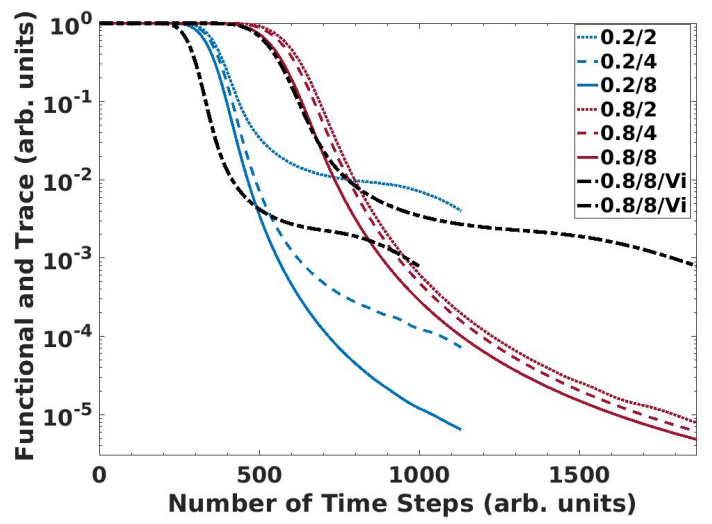

(b)

FIG. 7. (Color Online) (a) Imaginary potential corresponding to picture frame PML Eqs. (56) and (57) for $W=2(\Delta / 2)$, $V=0.8 \mathrm{eV}$. (b) Time evolution under scheme GIEXP and time step $\Delta_{t}^{E X P}$ under variation of frame width $W$ and $V_{o}$ : dotted lines: $W=2(\Delta / 2)$, dashed lines: $W=4(\Delta / 2)$, solid lines: $W=8(\Delta / 2) ; V_{o}=0.2 \mathrm{eV}$ : blue lines ending after 1132 time steps, $V_{o}=0.8 \mathrm{eV}$ : red lines ending after 1867 time steps. IPM simulations for $W=8(\Delta / 2)$ and $V_{o}=0.8 \mathrm{eV}$ : black dot-dashed lines.

require merely 1500 time steps. After less than 500 time steps the wave packet reaches the right boundary of the simulation region and suffers absorption and trapping, under virtually no back reflection. Decreasing values for $V_{o}$ under periodic BCs eventually one observes increasing transmission to the opposite side of the simulation region.

For comparison we applied $V_{I}(x, y)$ within the IPM to observe significantly reduced absorption in conjunction with back-reflection. This can be seen in Fig. 6 where the two dot-dashed lines give results within the latter method for $V_{o}=0.5 \mathrm{eV}$ and $W=6(\Delta / 2)$ and $V_{o}=1.6 \mathrm{eV}$ and $W=8(\Delta / 2)$. Both cases lead to a single-impact suppression factor of $\leq 10^{-2}$. The pronounced nearly flat part between 400 and 800 time steps arises from reflected and transmitted wave contributions traveling in the interior domain. Here, final time is reached after 1000 time steps.

In summary, PML leads to significantly improved absorption behaviour over the IPM, however, at the price of an increased number of iterations. From the analysis of uniform layers in the previous chapter the reasons are readily identified. Pure damping within the IPM is independent of dispersion and as such superior to PML. However, PML combines damping and trapping, thereby, it clearly wins out in the suppression of unwanted back-reflection from graded simulation boundaries. Discrete transparent BCs have been formulated for the (1+1)D Dirac equation under constant potentials at the boundaries 21. For a given FDS they give the best results possible, being limited by numerical/round-off errors only. However, their application in higher space dimensions and/or under space-and timedependence at the boundaries becomes numerically highly expensive, if not unfeasible. PML, on the other hand, can be taylored to give sufficiently accurate results, can readily be extended to higher dimensions, and, as will be illustrated below, works well under space-time-dependent potentials at the simulation boundaries.

Next we explore the effects of the variation of PML width $W$ and $V_{o}$. For illustration we use the GIEXP scheme with $\Delta_{t}=\Delta_{t}^{E X P}$, use $V_{o}=0.2 \mathrm{eV}$ and $0.8 \mathrm{eV}$, and increase the layer width from $W=2(\Delta / 2), 4(\Delta / 2)$ to $8(\Delta / 2)$. Fig. 7(a) displays the imaginary potential $V_{I}(x, y)$ for $W=2(\Delta / 2)$ and $V_{o}=0.8 \mathrm{eV}$. Again we use $m=0.02 \mathrm{eV}$ and a simulation time of $t=1000 \Delta_{t}^{C F L}$. We set $E_{x}=E_{y}=0.01 \mathrm{eV}$, so that the Gaussian now propagates from the centre towards the upper right corner of the simulation region. The evolution of functional Eq. $\sqrt{23}$ is given in Fig. 7 (b). For $V_{o}=0.2 \mathrm{eV}$, simulations terminate after 1132 iterations and results are given in blue. For $V_{o}=0.8 \mathrm{eV}$ they terminate after 1867 steps and results are given in red. For comparison, results for two IPM simulations using $V_{o}=0.8$ $\mathrm{eV}, W=8(\Delta / 2), \Delta_{t}=\Delta_{t}^{C F L}$ and, respectively, $\Delta_{t}=\Delta_{t}^{E X P}$ are shown using black dot-dashed lines. For PML the use of a moderate $V_{o}=0.2 \mathrm{eV}$ requires more than one physical layer (corresponding to two simulation layers) for a single-impact suppression factor of less than 1 per cent. On the other hand, 4 or 8 simulation layers per interface give excellent results in conjunction with a moderate increase in the minimum number of time steps. If grid size is the limiting factor, larger $V_{o}$ values allow the use of reduced PML width, as seen for $V_{o}=0.8 \mathrm{eV}$. Here, an increase in layer thickness from $W=2(\Delta / 2)$ to $W=8(\Delta / 2)$ gives but a moderate further increase in absorption. IPM works well and rather independently from step size, allowing completion after 1000 iterations. The use of $\Delta_{t}=\Delta_{t}^{C F L}$ for the GIEXP scheme for $V_{o}=0.8 \mathrm{eV}$ indeed leads to instability. We found that initial damping down to about $10^{-5}$ after about 900 iterations is followed by divergence. 


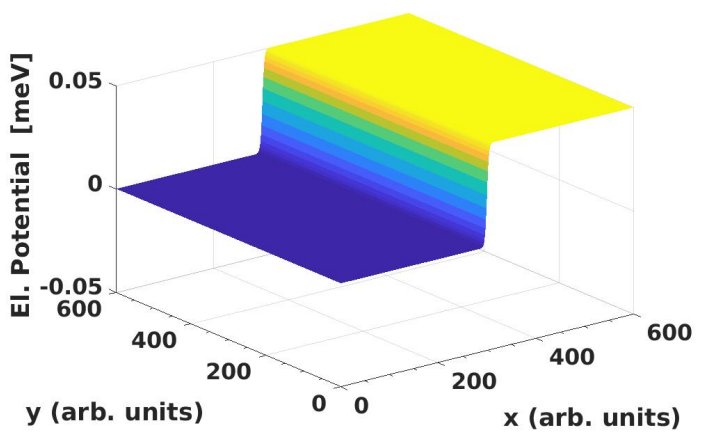

(a)

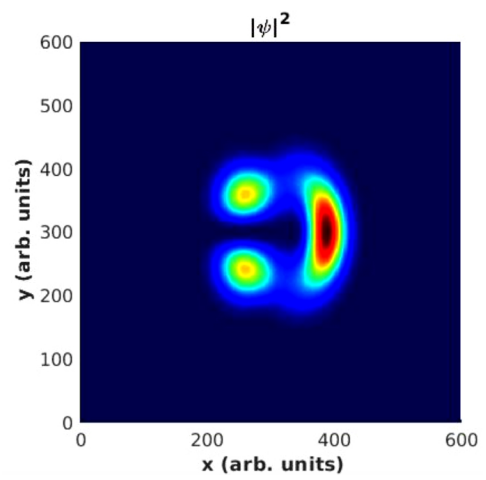

(c)

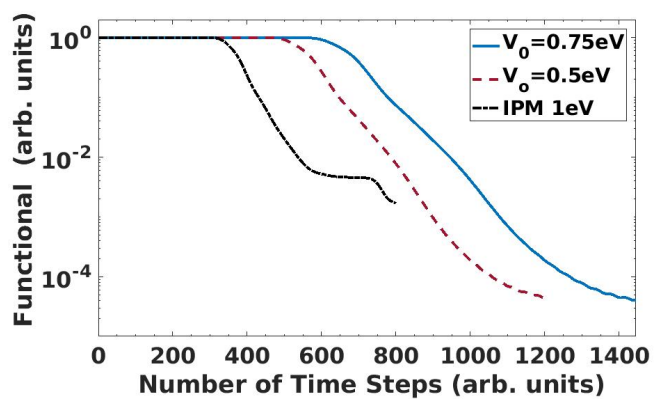

(b)

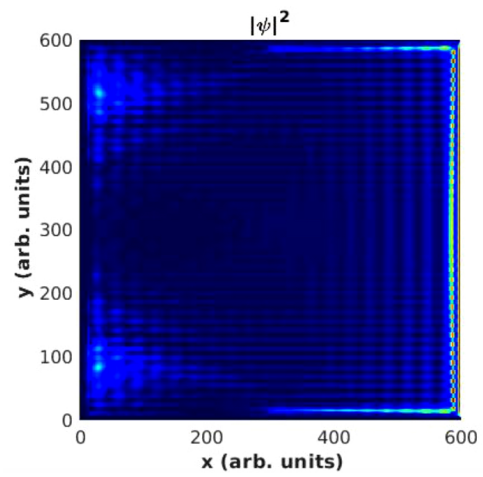

(d)

FIG. 8. (Color Online) Scattering at a static Klein step of height $V_{K}=0.05 \mathrm{eV}$, width $W_{K}=\Delta$ : (a) electric potential profile; (b) time-evolution of functional Eq. (23) under GIEXP for $V_{o}=0.75 \mathrm{eV}$ (solid blue line) and $V_{o}=0.5 \mathrm{eV}$ (dashed red line), and under IPM for $V_{o}=1 \mathrm{eV}$ (dot-dashed black line). Wave packet evolution under GIEXP $\left(V_{o}=0.5 \mathrm{eV}\right)$ : shortly after impact (c), at final time $(\mathrm{d})$.

\section{Particle propagation under discontinuous simulation boundaries}

Now we turn to a more challenging situation where, under periodic BCs, artificial discontinuities occur at the simulation boundaries. Such a situation arises, for example, when an electric bias is applied across the system. Since the concept of a group velocity across such a boundary is ill defined (unphysical), it is safer to join the PML formulation proposed above with zero - rather than periodic - BCs. Alternatively, one can set the group velocity across the boundary equal to zero, effectively, working with left- and right-sided group velocities.

First we shall consider a static Klein step $V(x, y)$, Fig. 8(a),

$$
V(x, y)=V_{K} \frac{1}{1+e^{\left(L_{x} / 2-x\right) / W_{K}}} .
$$

For $m=0$ Dirac fermions it provides a tunnel junction between particle and anti-particle states. On a $1 \mu m \times 1 \mu m$ simulation region of uniform mass $m=0 \mathrm{eV}$ we place a $600 \times 600$ grid for $u v$ storage and use zero BCs. The PML layer has a width of $\Delta$. We set $V_{K}=0.05 \mathrm{eV}$ and $W_{K}=\Delta$ and investigate a head-on impact by a Gaussian wave packet, $m=0, E_{x}=0.025 \mathrm{eV}, E_{y}=0$, width $25 \Delta$ released at grid position $\left(N_{x}=100, N_{y}=200\right)$. It's time evolution is investigated from time zero to $t=800 \Delta_{t}^{C F L}$. At the potential step the central part of the Gaussian is predominantly transmitted (spectral focussing), while the contributions with larger $k_{y}$ suffer partial reflection, shown in Fig. 8(c). The first main impact at the simulation boundary occurs at $t \approx 350 \Delta_{t}^{C F L}$. In Fig. 8(b) we compare the performance of the GIEXP scheme for $V_{o}=0.5 \mathrm{eV}$ and $V_{o}=0.75 \mathrm{eV}$ to that of the IMP for $V_{o}=1 \mathrm{eV}$. The IPM leads to a single impact damping down to less than one percent. The remaining wave contribution suffers back-reflection. It propagates almost freely across the interior domain, as evidenced by the near horizontal time evolution between impacts. In contrast, PML shows steady wave absorption to below $10^{-4}$ once the first impact has occurred, providing evidence for efficient suppression of back-reflection under PML. The wave distribution at final simulation time for $V_{o}=0.5 \mathrm{eV}$ is shown in Fig. $8(\mathrm{~d})$. Owing to the trapping action of PML, it is confined largely to the boundary region. 


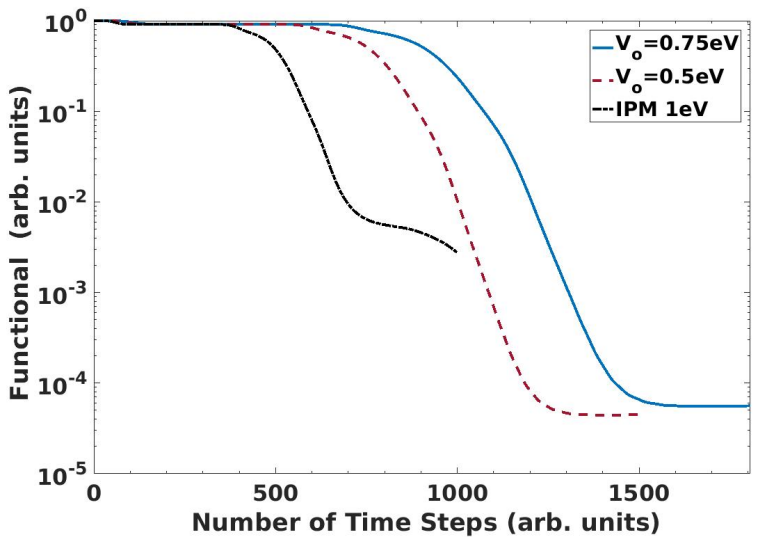

(a)

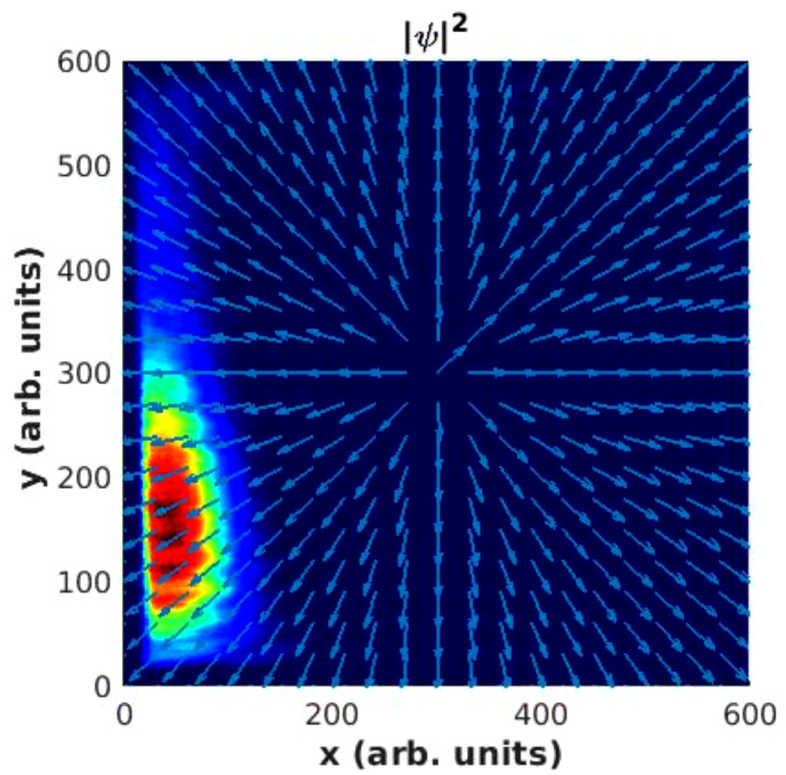

(c)

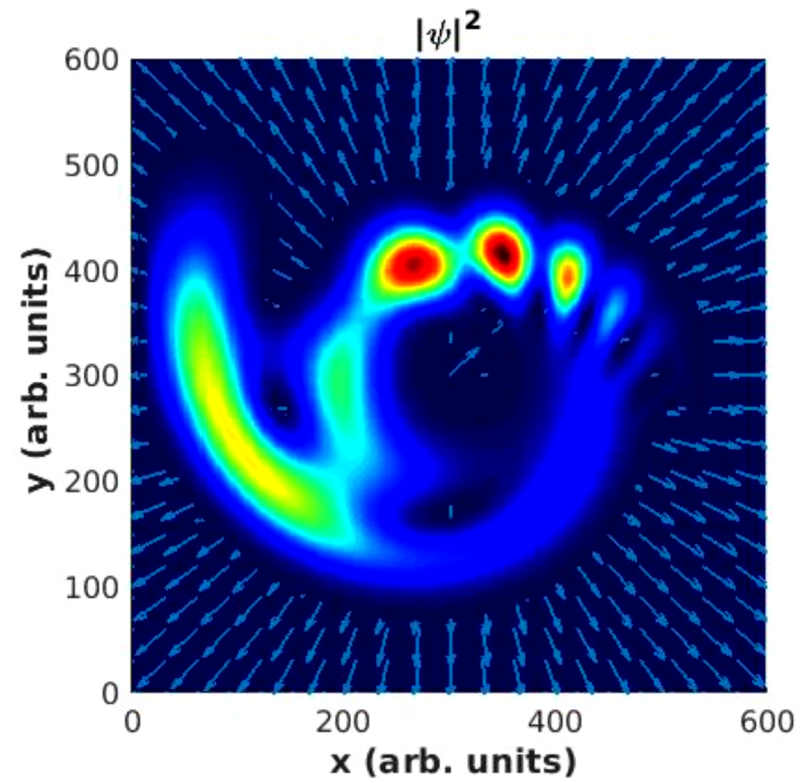

(b)

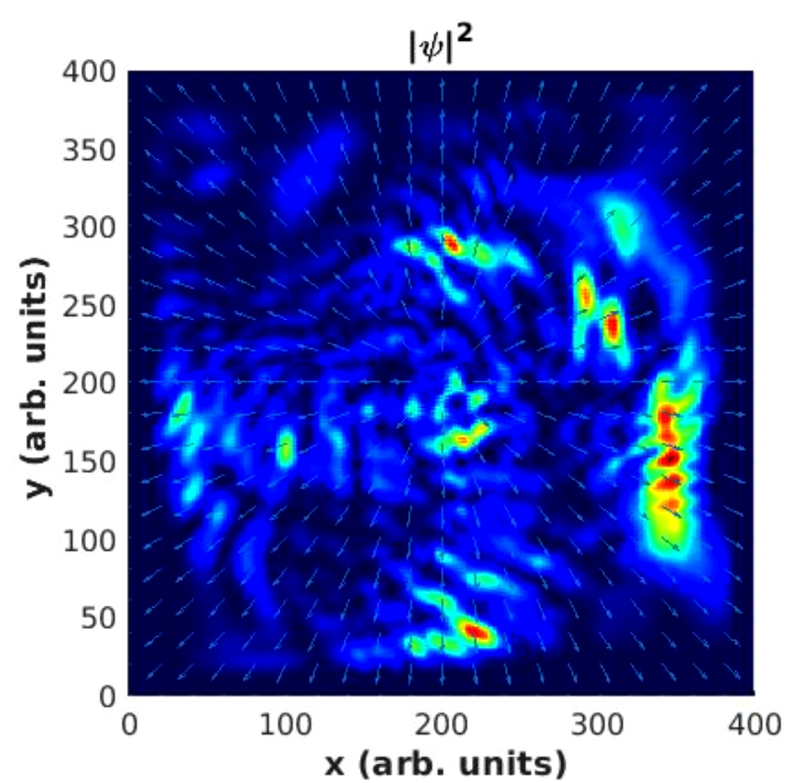

(d)

FIG. 9. (Color Online) Central collision of a Dirac fermion with a planar magnetic vortex $\left(\Phi_{o}=0\right)$ : (a) time-evolution of functional Eq. 23) under GIEXP for $V_{o}=0.75 \mathrm{eV}$ (solid blue line) and $V_{o}=0.5 \mathrm{eV}$ (dashed red line), and under IPM for $V_{o}=1 \mathrm{eV}$ (dot-dashed black line); wave packet prior to impact upon the boundaries (b), remnant wave packet under GIEXP (c), remnant wave packet under IPM (d).

For a second example of this kind we consider a planar ferromagnetic vortex

$$
\mathbf{M}(x, y)=M_{o}\left(\cos \left(\Phi_{o}+\nu \phi\right), \sin \left(\Phi_{o}+\nu \phi\right), 0\right), \phi=\arccos (y / x),
$$

coupling to the particle spin via the Pauli term

$$
H_{M}=\left(\begin{array}{cc}
0 & M_{x}-i M_{y} \\
M_{x}+i M_{y} & 0
\end{array}\right)
$$

in the Hamiltonian. $\mathbf{M}$ is the effective magnetisation of magnitude $M_{o}$. Phase $\Phi_{o}$ and integer core charge $\nu$ characterise the vortex type. For a $1 \mu \mathrm{m} \times 1 \mu \mathrm{m}$ simulation region for a Dirac fermion of mass $m$ zero we use a $600 \times 600 u v$ grid. 


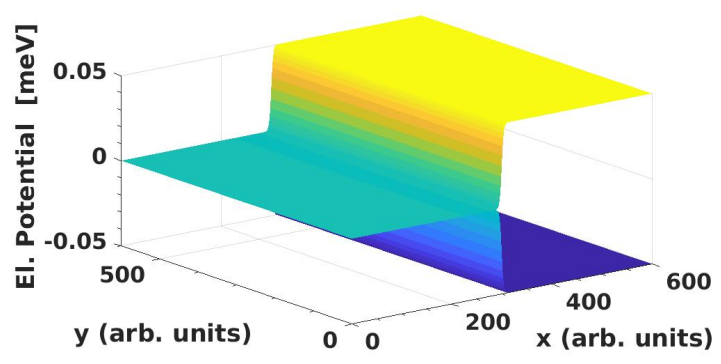

(a)

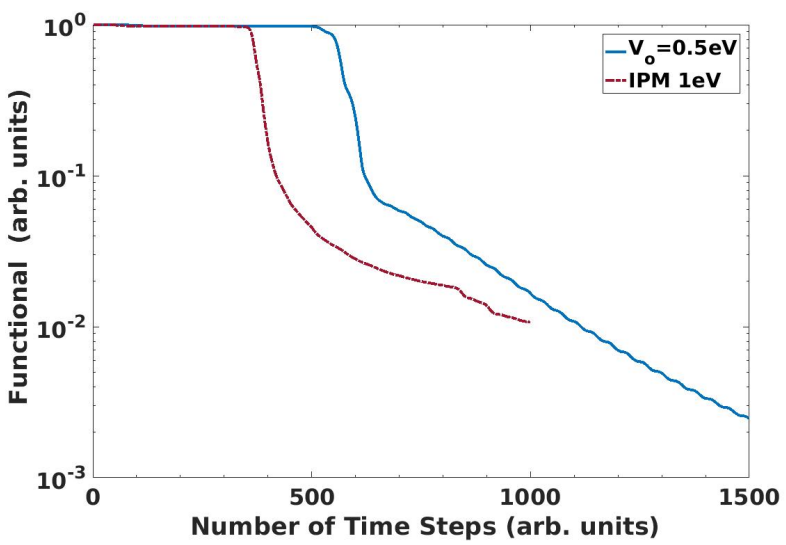

(b)

FIG. 10. (Color Online) Dynamic Klein step under the GIEXP and IPM scheme. Simulation parameters are given in the main text.

The eye of a planar vortex Eq. 59 with $M_{0}=0.03 \mathrm{eV}$ and $\nu=1$ is placed into the centre of the simulation region. Within GIEXP we investigate scattering of a Gaussian wave packet, $E_{x}=E_{y}=0.015 \mathrm{eV}$ and width $25 \Delta$ released at grid position $(100,100)$. The boundary layer widths $W$ is 4 grid positions. In Fig. 9 we show results for $\Phi_{o}=0$ (hedge-hog type), which turned out to be the most challenging type. Fig. 9(a) gives a comparison between the damping of functional Eq. 233 for $V_{o}=0.75$ and $V_{o}=0.50 \mathrm{eV}$ with damping under IPM for $V_{o}=1.00 \mathrm{eV}$. Fig. 9(b) gives the particle density upon collision with the vortex core region and prior to impact at the boundaries. The vortex is seen to apply a torque onto the incident particle and deflecting it in counter-clockwise direction. Thereby, the wave packet is spread out considerably before impinging upon the boundaries. Figs. 9.(c) and (d) give the remnant density at the respective final time step for time-evolution under GIEXP and IPM, respectively. For the former it is trapped near the boundary whereas, for the latter, remnant back-scattered wave contributions are found to be spread over the entire simulation region. All of the simulations give good results, however, GIEXP wins out by about two orders of magnitude. Simulations show that an ever increasing value for $V_{o}$ does not lead to improved damping. We know from the analysis above that large values of $V_{o}$ lead to trapping rather than damping. Thus a good balance between width $W$ and $V_{o}$ leads to optimal results. For the present case, an increase in $W$ would improve results further. Similar results have been found for vortices with $\Phi_{o}=\pi / 4$ and $\Phi_{o}=\pi / 2$.

\section{Numerical simulations for time-dependent textures}

PML based on the concept of group velocity bears an adiabatic character, in both space and time. Having established that PML can be adapted to abrupt potential changes (changes in the coefficients of the PDE, respectively, FDS) we now explore PML under time-dependent electromagnetic potentials at the boundary. This is relevant for transient transport analysis. For this purpose we first employ a time-dependent Klein step,

$$
V(x, y, t)=V_{K} \frac{1}{1+e^{\left(L_{x} / 2-x\right) / W_{K}}} \sin \left(\omega t+\phi_{V}\right) .
$$

We repeat the Klein step simulation from above and set $\omega=0.1 / \Delta_{t}^{C F L}, \phi_{V}=0$. Again we use the GIEXP PML scheme in conjunction with zero BCs. A comparison between evolution under GIEXP, $V_{o}=0.5 \mathrm{eV}$ and IPM $V_{o}=1$ $\mathrm{eV}$ is given in Fig. 10 which shows functional Eq. 23 versus time.

For final examples we study particle propagation in see-saw electric potentials for zero magnetisation and a hedgehog vortex sketched, respectively, in Fig. 111(a) and (b). The see-saw electric potential is $V(x, t)=0.05(x-$ $\left.L_{x} / 2\right) / L_{x} \sin (\omega t) \mathrm{eV}$. The oscillation frequency $\omega$ is $0.1 / \Delta_{t}^{C F L}$. The wave packet's initial conditions and the magnetic texture are the same as for the hedge-hog vortex investigation above. The time evolution for functional Eq. (23) for $M_{o}=0$ and $M_{o}=0.03 \mathrm{eV}$ are shown, respectively, in Figs. 11(c) and (d). While PML with $V_{o}=0.5 \mathrm{eV}$ (solid lines) clearly outperforms the IPM with $V_{o}=0.5 \mathrm{eV}$ (intermitted lines) in the first example, damping is comparable for the second case, where the IPM was applied for $\Delta_{t}=\Delta_{t}^{C F L}$, corresponding to 800 time steps, and the maximum time step under scheme GIEXP, corresponding to approximately 1200 time steps. In the latter example, the electromagnetic 


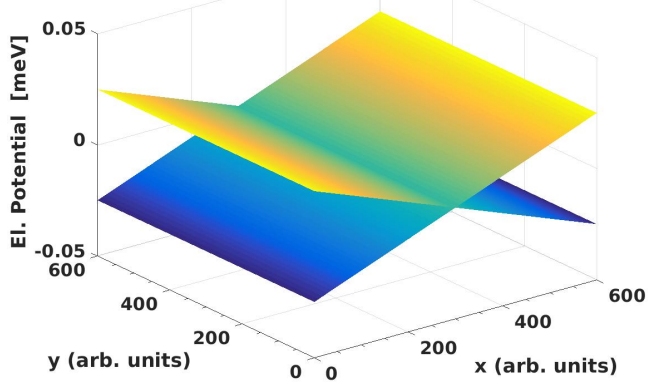

(a) See-saw potential.

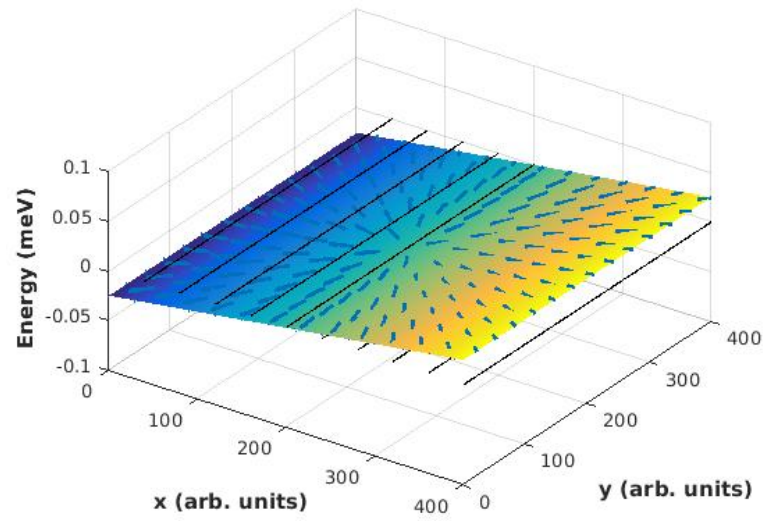

(b) Electromagnetic texture.

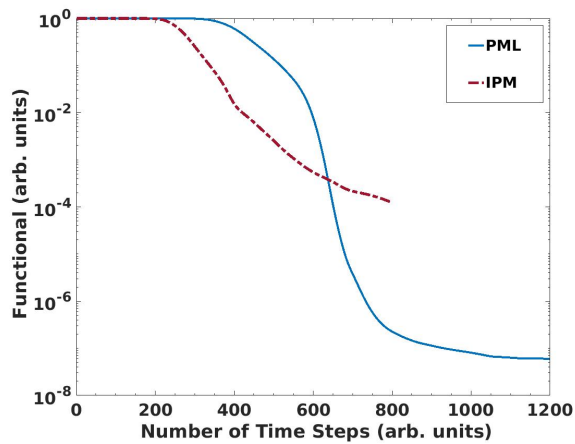

(c) Functional 23 versus time.

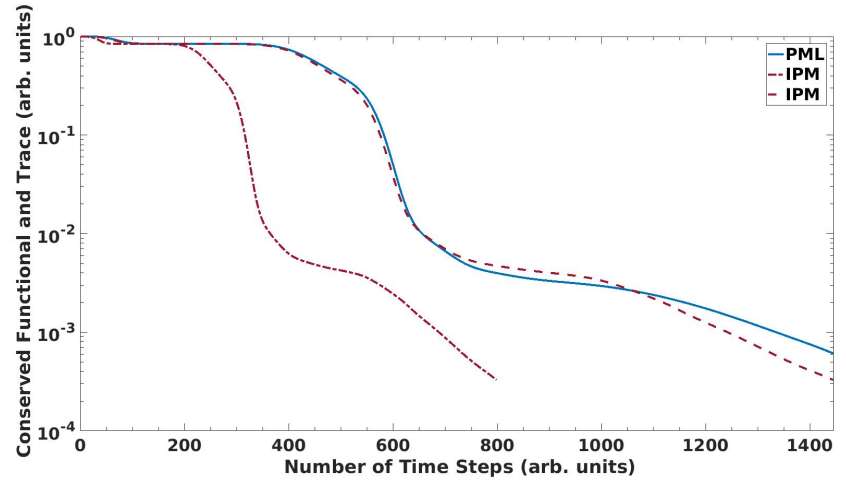

(d) Functional 23 versus time.

FIG. 11. (Color Online) Propagation of a Dirac fermion in see-saw potentials: (a) and (c) for zero magnetisation; (b) and (d) for a hedge-hog planar magnetic vortex.

texture leads to a prolonged trapping of wave packet components within the simulation region and therefore both methods lead to similar damping behaviour. In fact, whenever impact on the boundary or back-reflection from the simulation boundary is suppressed by the electro-magnetic texture itself, IPM and PML perform comparably well. The latter is the case, for example, when a chiral domain wall channel crosses the simulation boundary [22].

\section{SUMMARY AND CONCLUSIONS}

A general group-velocity-selective formulation of PML was applied to the Dirac equation in presence of space-timedependent electromagnetic potentials. PML extensions were derived for the $(2+1) \mathrm{D}$ PDE and two single-cone finite difference schemes (FDSs) of the latter. Several methods for the construction of PML auxiliary functions regarding both their integration in parallel and the incorporation of the electromagnetic fields were proposed. CFL conditions were derived for the FDS cases under PML. They are more stringent than the one for the original FDS and depend on the PML damping parameter(s), as well as the integration scheme for the PML auxiliary functions. Formulation was based on the $(2+1)$ D case with numerical examples geared towards topological insulator surface states. The presented approach, however, is readily applied to standard relativistic fermions in three space dimensions.

An investigation of PML-induced spectral changes for uniform layers was undertaken to demonstrate the proper damping behaviour for out-going wave contributions for both PDE and FDS. Selected basic numerical studies within uniform PML and picture-frame PML were performed to confirmed the latter. For moderate damping parameter, PML primarily leads to attenuation of out-going wave contributions. An increase of the former turns increased attenuation into trapping: a large PML damping parameter tends to flatten the energy dispersion in large portions of $k$-space. Further improvement can be achieved by an increase in the width of the absorbing layers. A layer width corresponding to about $4-6$ grid points (corresponding to about 1 percent of the sample length) was found to be 
sufficient.

Numerical tests performed under space- and time-dependent electromagnetic potentials have shown that the PML BCs hold up robustly, clearly outperforming the imaginary potential method regarding the overall absorption behaviour. PML is significantly more effective by a suppression of back-reflection in a combination of damping and trapping.

Competing with PML is the method of discrete transparent BCs (DTBCs) [12. It is based on BCs which feed in ghost values taken from analytic solutions for the exterior regions, for which constant in space- and time- PDE (FDS) coefficients are assumed to make this method tractable. BCs enter in form of convolutions in time. Thus the DTBC method invests in time whereas PML based on absorbing layers invests in space. The DTBC method is formally exact and limited only by round-off errors since the determination of growth factors as well as the required inverse Z-transform, in general, must be done numerically [21. This may be the reason why this method has, to our knowledge, by and large been limited to effective $(1+1) \mathrm{D}$ models only. Indeed we have applied this technique to the $(1+1) \mathrm{D}$ Dirac equation in the past to demonstrate its effectiveness [10]. One may expect that, under constant exterior conditions, it would work well in higher space dimensions, however, at a high numerical cost.

We note that a position- and time-dependent group velocity is an adiabatic concept. Therefore, abrupt potential discontinuities at simulation boundaries artificially arising from the use of periodic BCs should be avoided. The latter lead to unphysical group velocities across the boundaries which in turn can lead to numerical instability. In this case, zero BCs should be used in conjunction with PML. Remarkably, PML was found to be applicable to Dirac systems with time-dependent textures at and beyond the simulation boundaries. The latter are difficult to handle with spectral methods, such as the transparent BC approach [12]

Formulation was based on the $(2+1) \mathrm{D}$ case with numerical examples geared towards topological insulator surface states. The presented approach, however, is applicable to relativistic fermion simulations in three space dimensions as well.

\section{REFERENCES}

[1] P. A. M. Dirac, Proc. R. Soc. London A 117, 610 (1928).

[2] J. J. Sakurai, Advanced Quantum Mechanics (Addison-Wesley Publishing, 1967).

[3] W. N. Cottingham and D. A. Greenwood, An Introduction to the Standard Model of Particle Physics, 2nd ed. (Cambridge University Press, 2012).

[4] A. Maquet, R. Taïeb and V. Véniard, "Relativistic Laser-Atom Physics," (Springer, New York, NY, 2008) Chap. Strong Field Laser Physics pp 477-496.

[5] G. Rajasekaran, Reson. 8, 59 (2003).

[6] M. Z. Hasan and C. L. Kane, Rev. Mod. Phys. 82, 3045 (2010).

[7] K. Y. Y. Tokura and Tsukazaki, Nat. Rev. Phys. 1 1, 126 (2019).

[8] E. Raicher and S. Eliezer, Physical Review A 88, 022113 (2013).

[9] R. Hammer, W. Pötz, and A. Arnold, J. Comp. Phys. 265, 5070 (2014).

[10] R. Hammer, W. Pötz, and A. Arnold, J. Comp. Phys. 256, 728747 (2014).

[11] O. Pinaud, J. Comp. Phys. 289, 169180 (2015).

[12] X. Antoine, E. Lorin, and Q. Tang, Molecular Physics 115, 1861 (2017).

[13] X. Antoine and E. Lorin, J. Comp. Phys. 395, 583 (2019).

[14] E. X. Antoine, F. Fillion-Gourdeau and S. MacLean, J. Comp. Phys. 411, 109412 (2020).

[15] Walter Pötz, Phys. Rev. E 96, 053312 (2017).

[16] H. J. Rothe, Lattice Gauge Theories - An Introduction, Lecture Notes in Physics, Vol. 43 (World Scientific, 1992).

[17] J.-P. Bérenger, J. Comp. Phys. 114, 185 (1994).

[18] W. Pötz, Comp. Phys. Comm. 257, 107503 (2020).

[19] S. G. Johnson, "http://math.mit.edu/stevenj/18.369/pml.pdf," ((2010)).

[20] J. C. Strikwerda, Finite Difference Schemes and Partial Differential Equations (SIAM, 2004).

[21] Mathias Schwendt and Walter Pötz, Comp. Phys. Comm. 250, 107048 (2020).

[22] R. Hammer and W. Pötz, Phys. Rev. B 88, 235119 (2013). 